\title{
Error Bounds for Asymptotic Solutions of Differential Equations. II. The General Case ${ }^{1}$
}

\author{
Frank Stenger ${ }^{2}$
}

(May 26, 1966)

\begin{abstract}
The results of the preceding paper are extended to the general system of $n$ first-order differential equations having an irregular singularity of arbitrary rank at infinity. Formal solutions are explicitly constructed for the system in canonical form. Proofs of existence and uniqueness of solutions of integral equations defining the error are given. As an example, the case $n=2$ is solved completely, and a flow chart of the transformations of this case to canonical form is included.
\end{abstract}

\section{Introduction}

The system of differential equations under consideration in this paper is of the form

$$
\frac{d \mathbf{W}}{d z}=z^{h}\left[\sum_{k=0}^{s-1} \mathbf{A}_{k} z^{-k}+\mathfrak{A}_{s}(z) z^{-s}\right] \mathbf{W}, \quad s=0,1, \ldots,
$$

where $h$ is an integer and the matrices $\mathbf{A}_{k}$ and $\mathfrak{U}_{s}(z)$ are defined as in [1], except that no assumptions are made concerning eigenvalues of the matrices $\mathbf{A}_{k}(k=0,1, \ldots)$. We shall simultaneously consider the case of a regular point, the case of a regular singular point, and the case of an irregular singular point at infinity.

From the point of view of applications it is worthwhile recalling that when the eigenvalues of the lead coefficient matrix $\mathbf{A}_{0}$ are not distinct they can be made distinct by an arbitrarily small change in the elements of $\mathbf{A}_{0}$. The results of $[1]^{3}$ can then be applied, and the very complicated procedure given in this paper for obtaining a formal solution of (1.1) avoided. Indeed the existence of a linearly independent set of $n$ formal solution vectors for the system (1.1) was fully established only in 1937 ([2]) and more explicitly in 1955 ([3]).

We have not been able to improve on the method of [3] for the reduction of (1.1) to canonical form. We thus start with the canonical form given in [3] and establish a minor variation of it which enables us more easily to obtain error bounds. Next we explicitly construct formal solutions for the system in canonical form. Integral equations are then set up for the error vector, that is, for the difference between the partial sum of a formal solution vector and an actual solution vector.

As in [1], it is again possible to express the error by one or, at most, two Volterra vector integral equations. As opposed to [1], however, it is not always possible to choose the end points of integration in the Volterra integral equations to be at infinity.

\section{The Canonical Form}

The following theorem due to H. L. Turrittin [3] concerns transformation to canonical form, of the system (1.1), with eigenvalues of $\mathbf{A}_{0}$ not necessarily distinct. In this theorem the normalizing, exponential, zero-inducing, root-equalizing, and shearing transformations are defined as in [3].

\footnotetext{
${ }^{1}$ An invited paper based on the author's Ph. D thesis, University of Alberta (1965).

${ }^{2}$ Present address: University of Michigan, Ann Arbor, Mich.

${ }^{3}$ Figures in brackets indicate the literature references at the end of this paper.
} 
An example of each of these transformations will be given in our treatment of the special $n=2$ case in section 4 .

THEOREM 2.1: Let $h$ be an integer and

$$
\frac{\mathrm{d} \mathbf{X}}{\mathrm{dt}}=\mathrm{t}^{\mathrm{h}} \mathfrak{A}(\mathrm{t}) \mathbf{X}
$$

a system of differential equations in which

$$
\mathfrak{U}(\mathrm{t})=\sum_{\mathrm{k}=0}^{\mathrm{s}-\mathrm{i}} \mathbf{A}_{\mathrm{k}} \mathrm{t}^{-\mathrm{k}}+\mathfrak{A}_{\mathrm{s}}(\mathrm{t}) \mathrm{t}^{-\mathrm{s}} \quad(\mathrm{s}=0,1, \ldots)
$$

where the $\mathbf{A}_{\mathrm{k}}$ are constant $\mathrm{n} \times \mathrm{n}$ matrices and the elements of $\mathfrak{A}_{\mathrm{s}}(\mathrm{t})(\mathrm{s}=1,2, \ldots$. .) are holomorphic and uniformly bounded in some domain $\mathscr{D}_{\mathrm{t}}$ extending to infinity. Then the system (2.1) can be transformed by a finite sequence of normalizing, zero-inducing, root-equalizing and shearing transformations to the canonical system

$$
\frac{\mathrm{d} \mathbf{W}}{\mathrm{dz}}=\mathrm{z}^{\mathrm{r}} \mathfrak{S}(\mathrm{z}) \mathbf{W}
$$

where $\mathrm{z}=\mathrm{t}^{\mathrm{p}}, \mathrm{r}$ and $\mathrm{p}>0$ are integers,

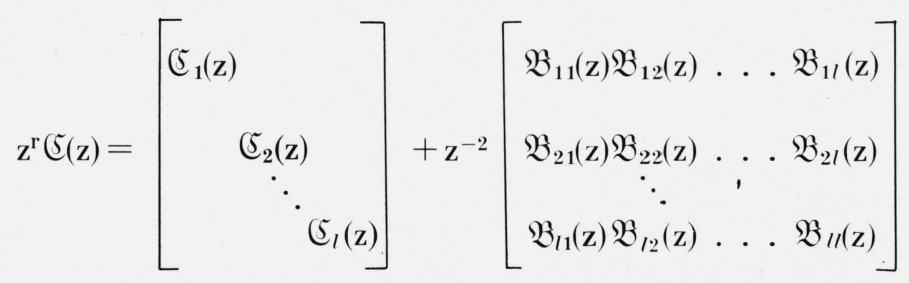

and

$$
\begin{aligned}
\mathrm{z}^{\mathrm{r}} \mathfrak{S}_{\mathrm{i}}(\mathrm{z}) & =\sum_{\mathrm{k}=0}^{\mathrm{r}+1} \mathbf{C}_{\mathrm{ik}} \mathrm{z}^{\mathrm{r}-\mathrm{k}} \mathbf{I}_{\mu_{\mathrm{i}}}+\mathrm{z}^{-1} \mathbf{J}_{\mu_{\mathrm{i}}} \\
\mathrm{i} & =1,2, \ldots ., \quad \mu_{1}+\mu_{2}+\ldots .+\mu_{l}=n \\
\mathfrak{B}_{\mathrm{ij}}(\mathrm{z}) & =\sum_{\mathrm{k}=0}^{\mathrm{s}-1} \mathbf{B}_{\mathrm{ijk}} \mathrm{z}^{-\mathrm{k}}+\mathfrak{B}_{\mathrm{ijs}}(\mathrm{z}) \mathrm{z}^{-\mathrm{s}},
\end{aligned}
$$

The elements of the matrices $\mathfrak{B}_{\mathrm{ij}}(\mathrm{z})$ are holomorphic for $\mathrm{z}$ in the domain $\mathscr{D}$ which corresponds to $\mathscr{D}_{\mathrm{t}}$ for all $\mathrm{z}$ sufficiently large. Each element of $\mathfrak{B}_{\mathrm{ijs}}(\mathrm{z})(\mathrm{i}, \mathrm{j}=1,2, \ldots, l ; \mathrm{s}=0,1, \ldots)$ is uniformly bounded on any path $\mathscr{P}$ extending to $\infty$ in $\mathscr{D}$. If $\mathrm{r} \leqq-2$ the matrices $\mathfrak{S}_{\mathrm{i}}(\mathrm{z})(\mathrm{i}=1,2, \ldots l)$ are identically zero. If $\mathrm{r}>-2$, the $\mathbf{I}_{\mu_{\mathrm{i}}}$ are $\mu_{\mathrm{i}} \times \mu_{\mathrm{i}}$ unit matrices, and the $\mathbf{J}_{\mu_{\mathrm{i}}}$ are $\mu_{\mathrm{i}} \times \mu_{\mathrm{i}}$ square matrices with zeros or ones or a mixture of zeros and ones in the first subdiagonal while all other elements of $\mathbf{J}_{\mu_{1}}$ are zero. No two of the matrices $\mathfrak{S}_{\mathrm{i}}(\mathrm{z})(\mathrm{i}=1,2, \ldots, l)$ in $(2.5)$ are identical. In particular, if $\mathrm{i} \neq \mathrm{j}$ and $\mathrm{c}_{\mathrm{ik}}=\mathrm{c}_{\mathrm{jk}}$ for $\mathrm{k}=0,1, \ldots, \mathrm{r}$, then corresponding to any particular fixed $\mathrm{j}(\mathrm{j}=1,2, \ldots, l)$ we have $-1 \leqq \operatorname{Rec}_{\mathrm{j}, \mathrm{r}+1}<0,0 \leqq \operatorname{Rec}_{\mathrm{i}, \mathrm{r}+1}<1$ and $\mathrm{c}_{\mathrm{i}, \mathrm{r}+1}-\mathrm{c}_{\mathrm{j}, \mathrm{r}+1} \neq 1 \quad(\mathrm{i}=1,2, \ldots, l ; \mathrm{i} \neq \mathrm{j})$.

The statement of the above theorem is different in two aspects from that given by Turrittin in [3]. In the first place Turrittin proves the theorem for the case when $\mathfrak{A}(\mathrm{t})$ has a convergent power series expansion about $t=0$. Secondly, Turrittin's version of the last sentence of the theorem is: In particular, if $\mathrm{i} \neq \mathrm{j}$ and $\mathrm{c}_{\mathrm{ik}}=\mathrm{c}_{\mathrm{jk}}$ for $\mathrm{k}=0,1, \ldots, \mathrm{r}$ then $\mathrm{c}_{\mathrm{i}, \mathrm{r}+1}-\mathrm{c}_{\mathrm{j}, \mathrm{r}+1}$ is not only not zero but is also not an integer. 
It is readily verified that all of the transformations: normalizing, zero-inducing, root-equalizing, and shearing preserve holomorphy of $\mathfrak{A}(t)$ for all $t$ sufficiently large, and $t \in \mathscr{D}_{t}$ extending to infinity.

We now establish the statement in the last sentence of Theorem 2.1, assuming Turrittin's version of the theorem. Consider the subset of integers $\{i\}$ of the set $\{i=1,2, \ldots ., l\}$ such that with $i \in\{i\}$, and $i \neq j, c_{i k}-c_{j k}=0, k=0,1, \ldots ., r$. Corresponding to the points $c_{i, r+1}$ in the complex $\lambda$ plane, we define integers $k_{i}$ as follows: if $c_{i, r+1}$ lies in the strip $k_{i} \leqq \operatorname{Re} \lambda<k_{i}+1$ we associate the integer $k_{i}$ with $c_{i, r+1}$. Without loss of generality we assume that $\{i\}=\{1,2, \ldots, \omega\}$ and that $k_{1} \leqq k_{2} \leqq \ldots . \leqq k_{\omega}$. Let $\kappa=k_{\omega}-k_{1}$.

We apply the sequence of zero-inducing transformations

$$
\mathbf{Z}_{k+1}=\left(\mathbf{I}+\mathbf{T}_{k} z^{-k}\right) \mathbf{Z}_{k} ; \quad k=1,2, \ldots, \kappa+1 ; \quad \mathbf{Z}_{1}=\mathbf{W}
$$

to (2.3), where $\mathbf{T}_{k}$ is an $n \times n$ matrix with an arbitrary leading $\omega \times \omega$ block and zeros everywhere else. Thus for example if

$$
\frac{d \mathbf{Z}_{k-1}}{d z}=z^{r}\left[\mathbf{C}_{0}+\mathbf{C}_{1} z^{-1}+\ldots .+\mathbf{C}_{r+1} z^{-r-1}+\mathbf{B}_{0} z^{-r-2}+\ldots .+\mathbf{B}_{k} z^{-k-r-1}+\ldots\right] \mathbf{Z}_{k-1}
$$

where the leading $\omega \times \omega$ blocks of $\mathbf{B}_{0}, \mathbf{B}_{1}, \ldots, \mathbf{B}_{k-1}$ have already been filled with zeros, then

$$
\frac{d \mathbf{Z}_{k}}{d z}=z^{r}\left[\mathbf{C}_{0}+\mathbf{C}_{1} z^{-1}+\ldots+\mathbf{C}_{r+1} z^{-r-1}+\mathbf{B}_{0} z^{-r-2}+\ldots+\mathbf{B}_{k}^{*} z^{-k-r-1}+\ldots\right] \mathbf{Z}_{k}
$$

where

$$
\mathbf{B}_{k}^{*}=\mathbf{B}_{k}-\mathbf{T}_{k} \mathbf{C}_{r+1}+\mathbf{C}_{r+1} \mathbf{T}_{k}+k \mathbf{T}_{k} .
$$

We divide the leading $\omega \times \omega$ blocks of the constant matrices $\mathbf{B}_{k}^{*}, \mathbf{B}_{k}, \mathbf{T}_{k}$ and $\mathbf{C}_{r+1}$ into smaller subblocks corresponding to the way in which $\mathbf{B}_{0}$ was subdivided in the above theorem. We denote these subblocks respectively by $\mathbf{B}_{i j k}^{*}, \mathbf{B}_{i j k}, \mathbf{T}_{i j k}$, and $\mathbf{C}_{i j, r+1}$. Equation (2.9) for $\mathbf{B}_{i j k}^{*}$ then becomes

$$
\mathbf{B}_{i j k}^{*}=\mathbf{B}_{i j k}+\left(\mathbf{C}_{i} \mathbf{I}_{\mu_{i}}+\mathbf{J}_{\mu_{i}}\right) \mathbf{T}_{i j k}-\mathbf{T}_{i j k}\left(\mathbf{C}_{j j} \mathbf{I}_{\mu_{j}}+\mathbf{J}_{\mu_{j}}\right)+k \mathbf{T}_{i j k}
$$

where $\mathbf{C}_{i}=\mathbf{C}_{i, r+1}$. Assuming Turrittin's version of Theorem 1 , we have $\lambda_{j}-\lambda_{i} \neq k$. Elements of $\mathbf{T}_{i j k}$ can therefore be computed ${ }^{4}$ starting from the right-hand vector, calculating from top to bottom and in this manner successively computing the vectors from right to left such that $\mathbf{B}_{i j k}^{*}=\mathbf{0}$. It is clear that each leading $\omega \times \omega$ block of $\mathbf{B}_{k}(k=0,1, \ldots, \kappa)$ can be filled with zeros in this manner.

The root-equalizing transformations

$$
\mathbf{Z}=\left[\begin{array}{ccc}
z^{k 1} \mathbf{I}_{\mu_{1}} & & \\
& & \\
z^{k 2} \mathbf{I}_{\mu_{2}} \\
& \ddots \\
& & \\
& & z^{k} \mathbf{I}_{\mu_{\omega}}
\end{array}\right] \mathbf{Y}, \quad \mathbf{Y}=\left[\begin{array}{ccc}
\mathbf{I}_{\mu_{1}} & \\
\ddots & \\
& \mathbf{I}_{\mu_{i-1}} & \\
& & z \mathbf{I}_{\mu_{i}} \\
& & \ddots \\
& & \mathbf{I}_{\mu_{\omega}}
\end{array}\right] \mathbf{W}
$$

are now applied to (2.8) above, changing $\mathbf{C}_{i, r+1}(i=1,2, \ldots, l)$ to the form described in the last sentence of Theorem 2.1.

${ }^{4}$ For further details, see [3] p. 33. 
The following theorem is also proved by Turrittin [3]. The main purpose of presenting a proof here is to obtain a recurrence relation for the coefficients of the solution matrix and to furnish an explicit algorithm for computing these coefficients.

THEOREM 2.2: The system of differential equations in the canonical form (2.3) to (2.5) possesses a formal independent series solution matrix of the form

$$
\tilde{\mathbf{W}}(\mathbf{z})=\tilde{\mathbf{U}}(\mathbf{z}) \mathbf{e}^{\mathfrak{Q}(\mathrm{z})}
$$

where

$$
\begin{gathered}
\tilde{\mathbf{U}}(\mathrm{z})=\left[\tilde{\mathbf{U}}_{\mathrm{ij}}(\mathrm{z})\right] \triangleq \sum_{\mathrm{k}=0}^{\infty} \mathbf{U}_{\mathrm{k}} \mathrm{z}^{-\mathrm{k}} \triangleq \sum_{\mathrm{k}=0}^{\infty}\left[\mathbf{U}_{\mathrm{ijk}}\right] \mathrm{z}^{-\mathrm{k}} \\
\tilde{\mathbf{U}}_{\mathrm{ij}}(\mathrm{z}) i s \mu_{\mathrm{i}} \times \mu_{\mathrm{j}}, \quad \mathbf{U}_{\mathrm{ij} 0}=\delta_{\mathrm{ij}} \mathbf{I}_{\mu_{\mathrm{i}}} \\
\preceq(\mathrm{z})=\left[\left(\mathbf{I}_{\mu_{\mathrm{i}}} \mathrm{q}_{\mathrm{i}}(\mathrm{z})+\mathbf{J}_{\mu_{\mathrm{i}}} \log \mathrm{z}\right) \delta_{\mathrm{ij}}\right]=\sum_{\mathrm{k}=0}^{\mathrm{r}} \frac{\mathbf{Q}_{\mathrm{k}}}{\mathrm{r}+\mathrm{l}-\mathrm{k}} \mathrm{z}^{\mathrm{r}+1-\mathrm{k}}+\mathbf{Q}_{\mathrm{r}+1} \log \mathrm{z} \\
\mathrm{q}_{\mathrm{i}}(\mathrm{z})=\sum_{\mathrm{k}=0}^{\mathrm{r}} \frac{\mathrm{q}_{\mathrm{ik}}}{\mathrm{r}+\mathrm{l}-\mathrm{k}} \mathrm{z}^{\mathrm{r}+1-\mathrm{k}}+\mathrm{q}_{\mathrm{i}, \mathrm{r}+1} \log \mathrm{z} \quad(\mathrm{i}, \mathrm{j}=1,2, \ldots, l) .
\end{gathered}
$$

Proof of Theorem 2.2: Let us put

$$
\begin{gathered}
z^{r}\left[\mathfrak{S}_{i}(z) \delta_{i j}\right]=\sum_{k=0}^{r+1} \mathbf{C}_{k} z^{r+1-k} \equiv z^{r} \mathbf{D}(z), \\
{\left[\mathfrak{B}_{i j}(z)\right]=\mathfrak{B}(z)=\sum_{k=0}^{s-1} \mathbf{B}_{k} z^{-k}+\mathfrak{B}_{s}(z) z^{-s} .}
\end{gathered}
$$

We substitute (2.11) into (2.3), and use the fact that $\cong$ and $\varrho^{\prime}$ commute, to find

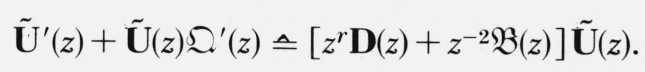

Expanding (2.3), using (2.12), (2.13), and (2.14) and equating equal powers of $z$, we obtain

$$
\sum_{k=0}^{\min (\mu, r+1)}\left(\mathbf{C}_{k} \mathbf{U}_{\mu-k}-\mathbf{U}_{\mu-k} \mathbf{C}_{k}\right)+(\mu-r-1) \mathbf{U}_{\mu-r-1}+\sum_{k=0}^{\mu-r-2} \mathbf{B}_{k} \mathbf{U}_{\mu-r-2-k}=\mathbf{O}
$$

for $\mu=0,1,2, \ldots$, where $\mathbf{U}_{k}=\mathbf{O}$ if $k<0$ and $\mathbf{C}_{k}=\mathbf{Q}_{k}=\mathbf{O}$ if $k>r+1$.

The proof of Theorem 2.2 will be complete once we prove the following lemma.

LEMMA 2.1: Under the assumption that $\mathfrak{\Omega}(\mathrm{z})$ has the form given by (2.12) with $\mathbf{U}_{0}=\mathbf{I}$, equation (2.16) uniquely determines $\mathbf{Q}_{\mu}(\mu=0,1,2, \ldots \mathrm{r}+1)$ and $\mathbf{U}_{\mu}(\mu=1,2,3, \ldots)$.

Proof of Lemma 2.1: The proof of the lemma is by induction. With $\mu=0,(2.16)$ gives

$$
\mathbf{C}_{0}-\mathbf{Q}_{0}=\mathbf{O}
$$

which has the unique solution $\mathbf{Q}_{0}=\mathbf{C}_{0}$. With $\mu=1,(2.15)$ becomes

$$
\mathbf{C}_{1}-\mathbf{Q}_{1}+\mathbf{C}_{0} \mathbf{U}_{1}-\mathbf{U}_{1} \mathbf{C}_{0}=\mathbf{O} .
$$


Now if $\mathbf{U}_{1}$ is an arbitrary $n \times n$ matrix we know from the definition of $\mathbf{C}_{0}$ that the $(i, i)$ th $(i=1,2$, $\ldots, l)$ blocks of $\mathbf{C}_{0} \mathbf{U}_{1}-\mathbf{U}_{1} \mathbf{C}_{0}$ consisting of $\mu_{i} \times \mu_{i}$ matrices are identically zero. Hence these blocks of $\mathbf{C}_{1}-\mathbf{Q}_{1}$ must also be zero, and therefore, $\mathbf{Q}_{1}=\mathbf{C}_{1}$. Furthermore, it follows that all $(i, j)$ th blocks of $\mathbf{U}_{1}$ for which $c_{i 0}-c_{j 0} \neq 0$ are uniquely determined and are zero. All $(i, j)$ th blocks of $\mathbf{U}_{1}$ for which $c_{i 0}=c_{j 0}$ cannot be determined at this point. In particular, it is not possible to solve for $\mathbf{U}_{i i 1}(i=1.2 \ldots . ., l)$.

Assume that for some integer $k, 0 \leqq k \leqq r$, we have completed solving (2.16) for all $\mu \leqq k$; that we have found $\mathbf{Q}_{\mu}=\mathbf{C}_{\mu}(\mu=0,1, \ldots, k)$ and that we have determined all blocks of the matrices $\mathbf{U}_{\mu}$ as far as possible. More precisely, we make the following assumptions for each $(i, j)$ th block of $\mathbf{U}_{\mu}(i, j=1,2, \ldots, l ; \mu=0,1, \ldots, k)$. If $c_{i \mu}-c_{j \mu}=0(\mu=0,1, \ldots, k-1)$ then assume $\mathbf{U}_{i j 1}, \mathbf{U}_{i j 2}, \ldots, \mathbf{U}_{i j k}$ are arbitrary, $\mu_{i} \times \mu_{j}$ matrices to be determined later. On the other hand, if $p$ is the smallest integer in $0 \leqq p \leqq k$ for which $c_{i p}-c_{j p} \neq 0$, we assume that $\mathbf{U}_{i j 0}$ $=\mathbf{U}_{i j 1}=\ldots=\mathbf{U}_{i j, k-p}=\mathbf{O}\left(\mu_{i} \times \mu_{j}\right)$, while $\mathbf{U}_{i j, k+1-p}, \ldots, \mathbf{U}_{i j k}$ are arbitrary $\mu_{i} \times \mu_{j}$ matrices.

For $\mu=k+1$, equation (2.16) becomes

$$
\mathbf{C}_{0} \mathbf{U}_{k+1}-\mathbf{U}_{k+1} \mathbf{C}_{0}+\ldots+\mathbf{C}_{k} \mathbf{U}_{1}-\mathbf{U}_{1} \mathbf{C}_{k}+\mathbf{C}_{k+1}-\mathbf{Q}_{k+1}=\mathbf{O}
$$

If $\mathbf{U}_{1}, \mathbf{U}_{2}, \ldots, \mathbf{U}_{k+1}$ are arbitrary $n \times n$ matrices, the $(i, i)$ th $(i=1,2, \ldots ., l)$ blocks of $\mathbf{C}_{\mu} \mathbf{U}_{k+1-\mu}$ $-\mathbf{U}_{k+1-\mu} \mathbf{C}_{\mu}$ are zero when $\mu=0,1, \ldots, k$. Hence these blocks of $\mathbf{C}_{k+1}-\mathbf{Q}_{k+1}$ must also be zero, and therefore $\mathbf{Q}_{k+1}=\mathbf{C}_{k+1}$. On incorporating this result in $(2.19)$ we find that the $(i, j)$ th block of (2.19) becomes

$$
\left(c_{i 0}-c_{j 0}\right) \mathbf{U}_{i j, k+1}+\left(c_{i 1}-c_{j 1}\right) \mathbf{U}_{i j, k}+\ldots+\left(c_{i k}-c_{j k}\right) \mathbf{U}_{i j 1}=\mathbf{O} .
$$

If $c_{i \mu}-c_{j \mu}=0, \mu=0,1,2, \ldots, k$, then we cannot solve for $\mathbf{U}_{i j 1}, \mathbf{U}_{i j 2}, \ldots, \mathbf{U}_{i j, k+1}$. We leave these matrices undetermined for the moment. On the other hand, if $p$ is the smallest nonnegative integer in $0 \leqq p \leqq k$ for which $c_{i p}-c_{j p} \neq 0$, then by the above induction hypothesis, (2.20) becomes $\left(c_{i p}-c_{j p}\right) \mathbf{U}_{i j, k+1-p}={ }_{i} \boldsymbol{O}\left(\mu_{i} \times \mu_{j}\right)$, and so under the assumption that $\mathbf{U}_{i j, k+2-p}, \ldots, \mathbf{U}_{i j, k+1}$ are arbitrary $\mu_{i} \times \mu_{j}$ matrices we have $\mathbf{U}_{i j, k+1-p}=\mathbf{O}$.

It is clear at this point that $\mathbf{Q}_{\mu}$ is uniquely determined for each $\mu=0,1, \ldots, r+1$. We may therefore write the $(i, j)$ th block of $(2.16)$ in the form

$$
\begin{aligned}
\sum_{k=0}^{r+1}\left(q_{i k}-q_{j k}\right) \mathbf{U}_{i j, \mu-k}+\mathbf{J}_{\mu_{i}} \mathbf{U}_{i j, \mu-r-1}- & \mathbf{U}_{i j, \mu-r-1} \mathbf{J}_{\mu_{j}} \\
& +(\mu-r-1) \mathbf{U}_{i j, \mu-r-1}+\sum_{k=0}^{\mu-r-2} \sum_{s=1}^{l} \mathbf{B}_{i s k} \mathbf{U}_{s j, \mu-r-2-k}=\mathbf{O} .
\end{aligned}
$$

For $\mu=r+2,(2.21)$ becomes

$$
\sum_{k=0}^{r+1}\left(q_{i k}-q_{j k}\right) \mathbf{U}_{i j, r+2-k}+\mathbf{J}_{\mu_{i}} \mathbf{U}_{i j 1}-\mathbf{U}_{i j 1} \mathbf{J}_{\mu_{j}}+\mathbf{U}_{i j 1}+\mathbf{B}_{i j 0}=\mathbf{O}
$$

If $q_{i \mu}-q_{j \mu}=0(\mu=0,1, \ldots, r)$, and $\mathbf{U}_{i j, r+2}$ is an arbitrary $\mu_{i} \times \mu_{j}$ matrix, then

$$
\left(1+q_{i, r+1}-q_{j, r+1}\right) \mathbf{U}_{i j 1}+\mathbf{J}_{\mu_{i}} \mathbf{U}_{i j 1}-\mathbf{U}_{i j 1} \mathbf{J}_{\mu_{j}}+\mathbf{B}_{i j 0}=\mathbf{O}
$$

where, under the assumptions of Theorem 2.1, $q_{i, r+1}-q_{j, r+1} \neq 1$ if $i \neq j$. In either case, whether $i=j$, or $i \neq j$, eq (2.23) is of the same form as (2.10) and hence it uniquely determines $\mathbf{U}_{i j 1}$. On the other hand, suppose that $p$ is the smallest integer in $0 \leqq p \leqq r$ for which $q_{i p}-q_{j p} \neq 0$. Then 
from the above analysis we know that $\mathbf{U}_{i j 0}=\mathbf{U}_{i j 1}=\ldots=\mathbf{U}_{i j, r+1-p}=\mathbf{O}\left(\mu_{i} \times \mu_{j}\right)$. Thus if $\mathbf{U}_{i j, r+3-p} \ldots, \mathbf{U}_{i j, r+2}$ are arbitrary $\mu_{i} \times \mu_{j}$ matrices, then

$$
\left(q_{i p}-q_{j p}\right) \mathbf{U}_{i j, r+2-p}+\mathbf{B}_{i j p}=\mathbf{O}
$$

and this equation uniquely determines the hitherto unspecified $\mathbf{U}_{i j, r+2-p}$. Thus we see that the $\mu_{i} \times \mu_{j}$ matrices $\mathbf{U}_{i j 1}$ are uniquely determined for $i, j=1,2, \ldots, l$, and hence that $\mathbf{U}_{1}$ has been completely and uniquely determined.

Assume that for some integer $k \geqq 0$ we have completed solving $(2.16)$ for $\mu=r+2, \ldots$, $r+2+k$; that we have uniquely determined $\mathbf{U}_{1}, \ldots, \mathbf{U}_{1+k}$ and that we have determined all blocks of the other matrices $\mathbf{U}_{1+s}, s>k$, as far as possible. More precisely, we make the following assumptions for each $(i, j)$ th block $\mathbf{U}_{i j, 1+\mu}$ of $\mathbf{U}_{1+\mu},(0 \leqq \mu \leqq r+2+k)$. If $q_{i \mu}-q_{j \mu}=0, \mu=0$, $1, \ldots, r$, then assume that $\mathbf{U}_{i j 1}, \ldots, \mathbf{U}_{i j, r+2+k}$ are arbitrary $\mu_{i} \times \mu_{j}$ matrices. On the other hand, if $p$ is the smallest nonnegative integer in $0 \leqq p \leqq r$ for which $q_{i p}-q_{j p} \neq 0$ then assume that $\mathbf{U}_{i j 1}, \ldots, \mathbf{U}_{i j, r+2+k-p}$ have been uniquely determined while $\mathbf{U}_{i j, r+3+k-p}, \ldots, \mathbf{U}_{i j, r+2}$ are arbitrary $\mu_{i} \times \mu_{j}$ matrices, to be determined later.

With $\mu=r+3+k,(2.21)$ becomes

$$
\sum_{s=0}^{r+1}\left(q_{i s}-q_{j s}\right) \mathbf{U}_{i j, r+3+k-s}+\mathbf{J}_{\mu_{i}} \mathbf{U}_{i j, 2+k}-\mathbf{U}_{i j, 2+k} \mathbf{J}_{\mu_{j}}+(k+2) \mathbf{U}_{i j, 2+k}+\sum_{s=0}^{k+1} \sum_{\nu=1}^{l} \mathbf{B}_{i \nu s} \mathbf{U}_{\nu j, k+1-s}=\mathbf{O} .
$$

If $q_{i s}-q_{j s}=0(s=0,1, \ldots, r)$, then with the assumption that $\mathbf{U}_{i j, r+3+k}$ is an arbitrary $\mu_{i} \times \mu_{j}$ matrix, the above induction hypothesis, and the assumptions of Theorem 2.1, we see that (2.25) is an equation in $\mathbf{U}_{i j, k+2}$ of exactly the same type as (2.10) was in $\mathbf{T}_{i j k}$, and thus uniquely determines $\mathbf{U}_{i j, k+2}$. On the other hand, suppose that $p$ is the smallest nonnegative integer in $0 \leqq p \leqq r$ for which $q_{i p}-q_{j p} \neq 0$. Then assuming $\mathbf{U}_{i j, r+4+k-p}, \ldots, \mathbf{U}_{i j, r+3+k}$ to be arbitrary $\mu_{i} \times \mu_{j}$ matrices, we see that eq (2.25) may be written

$$
\left(q_{i p}-q_{j p}\right) \mathbf{U}_{i j, r+3+k-p}=-\sum_{s=p+1}^{r+1}\left(q_{i s}-q_{j s}\right) \mathbf{U}_{i j, r+3+k-s}-(k+2) \mathbf{U}_{i j, k+2}-\sum_{s=0}^{k+1} \sum_{\nu=1}^{l} \mathbf{B}_{i \nu s} \mathbf{U}_{\nu j, k+1-s \cdot}
$$

By the induction hypothesis, all matrices on the right of (2.26) are known; hence $\mathbf{U}_{i j, r+3+k-p}$ is uniquely determined.

Thus the matrices $\mathbf{U}_{\mu}(\mu=1,2, \ldots$. .) are uniquely determined by eq (2.16).

The above proof can be easily modified for purposes of computing the $j$ th column of blocks only. Suppose we are interested in knowing the coefficients of a particular vector solution, say the $\nu$ th column $\widetilde{\mathbf{W}}_{j}^{\nu}(z)$ in the $j$ th column of blocks $\widetilde{\mathbf{W}}_{j}(z)$ of $\widetilde{\mathbf{W}}(z)$. If $\mathbf{J}_{\mu_{j}}$ has a one in its $\nu$ th column as well as in its $(\nu+1)$ th, . ., ( $\mu-1)$ th but not in its $\mu$ th column where $\mu \leqq \mu_{j}$, then it is readily shown that

$$
\widetilde{\mathbf{W}}_{j}^{\nu}(z) \hat{=} \sum_{k=0}^{\infty}\left\{\sum_{s=\nu}^{\mu} \mathbf{U}_{j k}^{s} \frac{(\log z)^{s-\nu}}{(s-\nu) !}\right\} z^{-k} e^{q_{j}(z)},
$$

where $\mathbf{U}_{j k}^{s}$ is the $s$ th $\left(s=1,2, \ldots, \mu_{j}\right)$ column vector in the $j$ th column of blocks of the matrix $\mathbf{U}_{k}$. Thus to obtain the $\nu$ th formal solution vector in the $j$ th column of blocks it is only necessary to compute the $\mu$ th, $(\mu-1)$ th, . . , $\nu$ th columns of $\mathbf{U}_{j k}$ at each stage. 


\section{Error Bounds for Formal Partial Sum Approximations to Actual Solutions}

We shall obtain an actual solution vector of the system (2.3) to (2.5) corresponding to $\nu$ th formal solution vector in the $j$ th column of blocks of formal solutions (eq (2.27)), and we shall obtain error bounds for the difference between a partial sum of (2.27) and the corresponding actual solution vector.

\subsection{The differential Equation for an Approximation}

Starting with the partial sum

$$
\Phi_{j m}(z)=\sum_{k=0}^{m-1} \mathbf{U}_{j k} z^{-k} e^{\curvearrowright_{j}(z)}
$$

where in the notation of Theorem 2.2, $\mathbf{U}_{j k}$ is the $j$ th column of blocks of the matrix $\mathbf{U}_{k}$ and

$$
\mathfrak{ح}_{j}(z)=q_{j}(z) \mathbf{I}_{\mu_{j}}+\mathbf{J}_{\mu_{j}} \log z
$$

we define $\mathbf{R}_{j m}(z)$ by

$$
\frac{d}{d z} \Phi_{j m}(z)-\left[\varrho^{\prime}(z)+z^{-2} \mathcal{L}(z)\right] \Phi_{j m}(z)=\mathbf{R}_{j m}(z) e^{\varrho_{j}(z)}
$$

On expanding the $(i, j)$ th block of (3.3) using (2.14) and (2.25) and denoting $q_{i}(z)-q_{j}(z)=q_{i j}(z)$, we obtain the following expression for $\mathbf{R}_{i j m}(z)$

$$
\begin{gathered}
q_{i j}^{\prime}(z) \mathbf{U}_{i j m} z^{-m}-\left[q_{i j}^{\prime}(z)-\left(q_{i p}-q_{j p}\right) z^{r-p}\right] \mathbf{U}_{i j m} z^{-m} \\
+\sum_{\mu=m+p+1}^{m+r+1}\left[\sum _ { s = \mu + 1 - m } ^ { \operatorname { m i n } ( \mu , r + 1 ) } \left(q_{i s}-q_{j s} \mathbf{U}_{i j, \mu-s}+\mathbf{J}_{\mu_{i}} \mathbf{U}_{i j, \mu-r-1}-\mathbf{U}_{i j, \mu-r-1} \mathbf{J}_{\mu_{j}}\right.\right. \\
\left.+(\mu-r-1) \mathbf{U}_{i j, \mu-r-1}+\sum_{s=0}^{\mu-r-2}\left\{\mathbf{B}_{s} \mathbf{U}_{j, \mu-r-2-s}\right\}_{i}\right] z^{r-\mu}-\sum_{s=0}^{m-1}\left\{\mathfrak{B}_{m-s}(z) \mathbf{U}_{j s}\right\}_{i} z^{-m-2}
\end{gathered}
$$

if $i \neq j$, and for some $p$ in $0 \leqq p \leqq r, q_{i p}-q_{j p} \neq 0$. On the other hand, if $q_{i p}=q_{j p}, 0 \leqq p \leqq r$, then

$$
\mathbf{R}_{i j m}(z)=\left[\left(q_{i, r+1}-q_{j, r+1}+m\right) \mathbf{U}_{i j m}+\mathbf{J}_{\mu_{i}} \mathbf{U}_{i j m}-\mathbf{U}_{i j m} \mathbf{J}_{\mu_{j}}\right] z^{-m-1}-\sum_{s=0}^{m-1}\left\{\mathfrak{B}_{m-s}(z) \mathbf{U}_{j s}\right\}_{i} z^{-m-2},
$$

where (3.5) includes the case $i=j$.

\subsection{The Various Possible Cases}

It is demonstrated in [4] that for each fixed $j(j=1,2, \ldots, l)$ the complete vicinity of infinity can be divided into a finite number $N$, say, of sectors $\mathscr{S}_{k j}(k=1,2, \ldots, N)$ such that for all $i$ $(i=1,2, \ldots, l)$ for which $q_{i p}-q_{j p} \neq 0$ for some integer $p$ in $0 \leqq p \leqq r$, we have either $\operatorname{Re} q_{i j}(z)$ bounded above, or else $\operatorname{Re} q_{i j}(z)$ tending to $+\infty$ as $z \rightarrow \infty$ in a particular sector $\mathscr{S}_{\mathrm{kj} \text {. This fact }}$ enables us to classify the various ways of constructing actual solution vectors corresponding to formal solution vectors under two different headings: (a) those cases for which it is possible to define an actual solution vector by a single Volterra integral equation; and (b) those cases for which we require a simultaneous pair of Volterra integral equations to define a solution vector.

Let us establish our notation for purposes of characterizing cases (a) and (b) above. The fixed limits of integration in the closure, $\overline{\mathscr{D}}$, of the domain $\mathscr{D}$ in which $\subseteq(z)$ is holomorphic will 
be denoted by $\{\zeta\}$. In case (a) $\{\zeta\}$ consists of a single point $\zeta$, say, and in case (b) $\{\zeta\}$ consists of two points, $\zeta_{1}$, and $\zeta_{2}$, say. $\mathscr{E}_{a}$ will denote a closed disc with centre at the origin and radius a. If for all integers $i$ in the range $1 \leqq i \leqq l q_{i p}=q_{j p}$ for $p=0,1, \ldots, r$, then $a=0$. provided that every member of $\{\zeta\}$ is unbounded. ,Put $\chi=\min |\zeta|$. If $\chi<\infty$ we set $a=\chi$. If, in case (b), all $\zeta \in\{\zeta\}$

members of $\{\zeta\}$ are bounded then we assume that $\zeta_{1} \neq \zeta_{2}$ and $\left|\zeta_{1}\right|=\left|\zeta_{2}\right|$. If, for at least one $i$ in the range $1 \leqq i \leqq l$, we have $q_{i p} \neq q_{j p}$ for some $p \in(0,1, \ldots, r)$, we let $a_{0}$ denote the maximum modulus of the set of zeros of $q_{i j}^{\prime}(z)$ for all such $i$, and set $a=a_{0}$ if $\chi=\infty$. If $\chi<\infty$ chose $\{\zeta\}$ in such a way that $\chi \geqq a_{0}$ and no member of $\{\zeta\}$ coincides with a zero of $q_{i j}^{\prime}(z)$. Let $\mathscr{D}^{*}=\mathscr{D}-\mathscr{E}_{a}-\mathscr{S}_{\text {where }}$ $\mathscr{S}$ is empty or $\{\infty\}$ according to whether or not $\mathscr{Q}(z)=\mathbf{O}$. The paths $\mathscr{P}$ are defined as in [1].

Case (a) above is related to the extreme eigenvalue case in [1]. It is characterized as follows. We define a region $\mathscr{D}(\mathrm{z}, \zeta)$ to be the union of all points $\mathrm{z}$, other than $\zeta$, such that there is a path $\mathscr{P}$ connecting $\mathrm{z} \in \mathscr{D}(\mathrm{z}, \zeta)$ and $\zeta$ satisfying the following extreme eigenvalue conditions:

(1) If $\zeta \in \bar{D}^{*}-\mathscr{D}^{*}$, then $\mathscr{P}$ lies entirely in $\mathscr{D}^{*}$ except for $\zeta$.

(2) For given $\zeta$ and $\mathrm{z}$, all elements of the matrices $\exp \left\{\Omega_{\mathrm{i}}(\tau)-\mathfrak{Q}_{\mathrm{i}}(\mathrm{t})-\mathbf{I}_{\mu_{\mathrm{i}}}\left[q_{\mathrm{j}}(\tau)-q_{\mathrm{j}}(\mathrm{t})\right]\right\}$ $(\mathrm{i}=1,2, \ldots, l: \mathrm{i} \neq \mathrm{j})$ are bounded for all points $\mathrm{t}, \tau$ in the order $\zeta, \mathrm{t}, \tau, \mathrm{z}$ on $\mathscr{P}$. If $\mathbf{J}_{\mu_{\mathrm{j}}} \neq \mathbf{O}$, or if for some $\mathrm{i}(\mathrm{i}=1,2, \ldots, l ; \mathrm{i} \neq \mathrm{j})$ we have $\mathrm{q}_{\mathrm{ip}}=\mathrm{q}_{\mathrm{jp}}$ for $\mathrm{p}=0,1, \ldots, \mathrm{r}$, we require that $\tau / \mathrm{t}$ be bounded.

(3) $V_{\mathscr{P}}\left(\mathrm{t}^{\eta-1}\right)$ is bounded, where the fixed number $\eta$ may be taken to be zero if $\mathbf{J}_{\mu_{\mathrm{j}}}=\mathbf{O}$ but otherwise $\eta$ is an arbitrary positive number less than 1 .

Case (b) above is related to the interior eigenvalue case in [1]. It is characterized as follows. We define a region $\mathscr{D}\left(\zeta_{1}, \zeta_{2}, \eta\right)$ to be the union of the points $\mathrm{z}$ for which there is a path $\mathscr{P}$ connecting $\zeta_{1}, \mathrm{z}$ and $\zeta_{2}$ (in that order) satisfying the following interior eigenvalue conditions:

(1) Except possibly for $\zeta_{1}$ and $\zeta_{2}, \mathscr{P}$ lies entirely in $\mathscr{D}^{*}$.

(2) The integers $\mathrm{i}=1,2, \ldots, l$ are subdivided into two nonempty disjoint classes $\mathbf{N}_{1}$ and $\mathbf{N}_{2}$ such that if $\zeta_{1}$, and $\zeta_{2}$ are given, then for all points $\mathrm{t}$ and $\tau$ in the order $\zeta_{1}, \mathrm{t}, \tau, \zeta_{2}$ on $\mathscr{P}$, the elements of the matrix $\mathfrak{S}_{\mathrm{ij}}(\tau, \mathrm{t})=\exp \left\{\mathfrak{\Omega}_{\mathrm{i}}(\tau)-\mathfrak{S}_{\mathrm{i}}(\mathrm{t})-\mathbf{I}_{\mu_{\mathrm{i}}}\left[q_{\mathrm{j}}(\tau)-q_{\mathrm{j}}(\mathrm{t})\right]\right\}$ are bounded if $\mathrm{i} \in \mathbf{N}_{1}(i \neq \mathrm{j})$ while if $\mathrm{i} \in \mathbf{N}_{2}(\mathrm{i} \neq \mathrm{j})$ ) then all elements of the matrix $\mathfrak{\Omega}_{\mathrm{ij}}(\mathrm{t}, \tau)$ are bounded. If $\mathbf{J}_{\mu_{\mathrm{j}}} \neq \mathbf{O}$, or if for some $\mathrm{i}$ in the range $(1, l) \quad(\mathrm{i} \neq \mathrm{j})$ we have $q_{i p}=q_{j p}$ for $\mathrm{p}=0,1, \ldots, \mathrm{r}$, then we assume without loss of generality that both $\mathrm{i}$ and $\mathrm{j}$ are in $\mathbf{N}_{2}$, that $\zeta_{1}$ is bounded, and that $\mathrm{t} / \tau$ remains bounded for all points $\mathrm{t}, \tau$ in the order $\zeta_{1}, \mathrm{t}, \tau, \zeta_{2}$ on $\mathscr{P}$.

(3) $\frac{1}{2}\left\{\mathrm{a}_{1}+\mathrm{a}_{4}+\sqrt{\left(\mathrm{a}_{1}-\mathrm{a}_{4}\right)^{2}+4 \mathrm{a}_{2} \mathrm{a}_{3}}\right\} \mathscr{V}_{\mathscr{P}}\left(\mathrm{t}^{\eta-1}\right)<1$, where $\eta$ may be taken zero if $\mathbf{J}_{\mu_{\mathrm{j}}}=\mathbf{O}$, but otherwise $\eta$ is an arbitrary positive number less than 1 , and $a_{1}, a_{2}, a_{3}$ and $a_{4}$ are defined by (3.31).

We observe that it is no longer always possible to choose the points $\{\zeta\}$ at infinity, in contrast to the cases in [1].

If $r \geqq 0$ we can again represent the eigenvalues of $\mathbf{C}_{0}$ as points in the complex plane, and enclose them by the smallest strictly convex closed polygon. As opposed to the distinct eigenvalue case it is now possible to have several formal solution vectors corresponding to a particular eigenvalue $q_{0 j}$ of $\mathbf{C}_{0}$. Nevertheless, if $q_{0 j}$ is an interior point of the polygon and if at least one endpoint of integration is taken at infinity ${ }^{5}$ it will be necessary to express the error vector by a simultaneous pair of Volterra vector integral equations. If $q_{0 j}$ is an extreme point of the polygon and only one formal vector solution corresponds to $q_{0 j}$, it is again possible, as in the distinct eigenvalue case, to express the error vector by a single Volterra vector integral equation. If $q_{0 j}$ is an extreme point of the polygon and more than one formal vector solution corresponds to $q_{0 j}$, or if $q_{0 j}$ is any other boundary point of the polygon, we can always determine whether we can express the error vector by a single or a simultaneous pair of Volterra integral equations by testing whether or not the above extreme eigenvalue conditions can be satisfied.

${ }^{5}$ We are here attempting to construct actual solutions for which the formal solutions are asymptotic expansions. 
We are now prepared to set up the integral equations. Let a vector $\mathbf{W}_{j}^{\nu}(z)$ of holomorphic functions satisfy the differential equation (2.3). Denoting the $\nu$ th column vector of $\Phi_{j m}$ by $\Phi_{j m}^{\nu}$, we find from (3.3) and (2.27) that the error vector

$$
\boldsymbol{\epsilon}_{j m}^{\nu}(z)=\left(\mathbf{W}_{j}^{\nu}(z)-\Phi_{j m}^{\nu}(z)\right) e^{-q_{j}(z)}
$$

satisfies the differential equation

$$
\frac{d}{d z} \boldsymbol{\epsilon}_{j m}^{\nu}(z)-\left[\mathbf{D}_{j}^{\prime}(z)+z^{-2} \mathfrak{B}(z)\right] \boldsymbol{\epsilon}_{j m}^{\nu}(z)=-\sum_{s=\nu}^{\mu} \mathbf{R}_{j m}^{s}(z) \frac{(\log z)^{s-\nu}}{(s-\nu) !}
$$

where $\mathbf{R}_{j m}^{s}(z)$ is the $s$ th column vector of $\mathbf{R}_{j m}(z)$, and $\mathbf{D}_{j}(z)=\preceq(z)-\mathbf{I} q_{j}(z)$.

Hence for the extreme eigenvalue case, if the vector $\boldsymbol{\epsilon}_{j m}^{\nu}(z)$ satisfies

$$
\boldsymbol{\epsilon}_{j m}^{\nu}(z)=\int_{\zeta}^{z} e^{\mathbf{D}_{\{z)}\left(\mathbf{D}_{f}(t)\right.} t^{-2 \mathfrak{B}(t) \boldsymbol{\epsilon}_{j m}^{\nu}(t) d t+\mathbf{R}_{j m}^{\nu *}(z)}
$$

where $\mathbf{R}_{j m}^{\nu *}(z)$ is defined by

$$
\mathbf{R}_{j m}^{\nu *}(z)=-\int_{\zeta}^{z} e^{\mathbf{D}_{j}(z)-\mathbf{D}_{j}(t)} \sum_{s=\nu}^{\mu} \mathbf{R}_{j m}^{s}(t) \frac{(\log t)^{s-\nu}}{(s-\nu) !} d t,
$$

and the path $\mathscr{P}$ of integration satisfies the above extreme eigenvalue conditions, then $\boldsymbol{\epsilon}_{j m}^{\nu}(z)$ simultaneously satisfies (3.7).

For the interior eigenvalue case we partition $\boldsymbol{\epsilon}_{j m}^{\nu}(z)$ into $\boldsymbol{\epsilon}_{j m 1}^{\nu}(z)$ and $\boldsymbol{\epsilon}_{j m 2}^{\nu}(z), \mathbf{D}_{j}(z)$ into $\mathbf{D}_{j 1}(z)$ and $\mathbf{D}_{j 2}(z)$ and $\mathbf{R}_{j m}^{s}$ into $\mathbf{R}_{j m 1}^{s}(z), \mathbf{R}_{j m 2}^{s}(z)$, and in a corresponding manner $\mathfrak{B}(z)$ into $\mathfrak{a}_{1}(z), \mathfrak{a}_{2}(z), \mathfrak{a}_{3}(z)$ and $a_{4}(z)$ so that (3.7) may be written

$$
\frac{d}{d z}\left(\begin{array}{c}
\boldsymbol{\epsilon}_{j m 1}^{\nu}(z) \\
\boldsymbol{\epsilon}_{j m 2}^{\nu}(z)
\end{array}\right)-\left[\left(\begin{array}{c}
\mathbf{D}_{j 1}^{\prime}(z) \mathbf{O} \\
\\
\mathbf{O} \mathbf{D}_{j 2}^{\prime}(z)
\end{array}\right)+z^{-2}\left(\begin{array}{cc}
a_{1}(z) & \mathfrak{a}_{2}(z) \\
a_{3}(z) & a_{4}(z)
\end{array}\right)\right] \quad\left(\begin{array}{c}
\boldsymbol{\epsilon}_{j m 1}^{\nu}(z) \\
\boldsymbol{\epsilon}_{j m 2}^{\nu}(z)
\end{array}\right)=-\sum_{s=\nu}^{\mu}\left(\begin{array}{c}
\mathbf{R}_{j m 1}^{s}(z) \\
\mathbf{R}_{j m 2}^{s}(z)
\end{array}\right) \frac{(\log z)^{s-\nu}}{(s-\nu) !}
$$

In (3.10) $\boldsymbol{\epsilon}_{j m 1}^{\nu}(z)$ contains all the $i$ th elements of $\boldsymbol{\epsilon}_{j m}^{\nu}(z)$ for which $i \in \mathbf{N}_{1}$, and $\boldsymbol{\epsilon}_{j m 2}^{\nu}(z)$ contains all the $i$ th elements for which $i \in \mathbf{N}_{2}$. It follows that if $\boldsymbol{\epsilon}_{j m}^{\nu}(z)$ satisfies

$$
\left(\begin{array}{c}
\boldsymbol{\epsilon}_{j m 1}^{\nu}(z) \\
\boldsymbol{\epsilon}_{j m 2}^{\nu}(z)
\end{array}\right)=\left(\begin{array}{cc}
\int_{\zeta_{1}}^{z} & \mathbf{0} \\
\mathbf{0} & \int_{\zeta_{2}}^{z}
\end{array}\right)\left[\left(\begin{array}{cc}
e^{\mathbf{D}_{j 1}(z)-\mathbf{D}_{j 1}(t)} & \mathbf{O} \\
\mathbf{O} & e^{\mathbf{D}_{j 2}(z)-\mathbf{D}_{j(t}(t)}
\end{array}\right) t^{-2}\left(\begin{array}{cc}
\mathfrak{a}_{1}(t) & a_{2}(t) \\
a_{3}(t) & \left.a_{4}(t)\right)
\end{array}\right)\left(\begin{array}{c}
\boldsymbol{\epsilon}_{j m 1}^{\nu}(t) \\
\boldsymbol{\epsilon}_{j m 2}^{\nu}(t)
\end{array}\right)\right] d t+\left(\begin{array}{c}
\mathbf{R}_{j m 1}^{\nu *}(z) \\
\mathbf{R}_{j m 2}^{\nu *}(z)
\end{array}\right)
$$

where

$$
\mathbf{R}_{j m k}^{\nu *}(z)=-\int_{\zeta_{k}}^{z} e^{\mathbf{D}_{j k}(z)-\mathbf{D}_{j k}(t)} \sum_{s=\nu}^{\mu} \mathbf{R}_{j m k}^{s}(t) \frac{(\log t)^{s-\nu}}{(s-\nu) !} d t, k=1,2,
$$

and the path of integration satisfies the above interior eigenvalue conditions, then $\boldsymbol{\epsilon}_{j m}^{\nu}(z)$ also satisfies (3.7). 


\subsection{Treatment of $\mathbf{R}_{i j m}(z)$}

We shall derive expressions for the elements of the matrices (3.9) and (3.12) which will enable us to obtain good bounds in the following sections. Let us consider the block ${ }^{6}$

$$
\mathbf{R}_{i j m}^{*}(z)=-\int_{\zeta}^{z} e^{\Omega_{\lambda}(z)-\Omega_{i}(t)} \mathbf{R}_{i j m}(t) e^{\varrho_{j}(t)-q_{j}(z)} d t .
$$

We split (3.13) into its dominant part plus a remainder; thus we express $\mathbf{R}_{i j m}(t)$ in the form

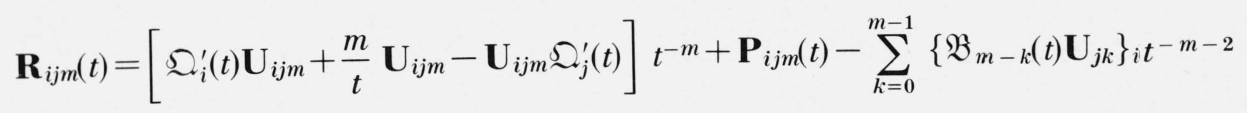

where

$$
\begin{aligned}
& \mathbf{P}_{i j m}(t)=-\left[\left[\left(q_{i, p+1}-q_{j, p+1}\right) t^{r-p-1}+\ldots .+\left(q_{i, r+1}-q_{j, r+1}\right) t^{-1}+m t^{-1}+\mathbf{J}_{\mu_{i}} t^{-1}\right] \mathbf{U}_{i j m}-U_{i j m} \mathbf{J}_{\mu_{j}} t^{-1}\right] t^{-m} \\
& \underset{\mu=m+p+1}{m+r+1}\left[\sum_{k=\mu+1-m}^{\min }\left(q_{i k}-q_{j k}\right) \mathbf{U}_{i j, \mu-k}+\mathbf{J}_{\mu_{i}} U_{i j, \mu-r-1}-U_{i j, \mu-r-1} \mathbf{J}_{\mu_{j}}\right. \\
& \left.+(\mu-r-1) U_{i j, \mu-r-1}+\sum_{k=0}^{\mu-r-2}\left\{B_{k} U_{j}, \mu-r-2-k\right\}_{i}\right] t^{r-\mu} .
\end{aligned}
$$

The form of the first term in (3.14) is such that when substituted into (3.13) it can be readily integrated by parts. We define $\mathbf{S}_{i j}(z, t)$ by

$$
\mathbf{S}_{i j}(z, t) e^{q_{j i}(z)-q_{i j}(t)}=e^{\wp_{i}(z)-\Sigma_{i}(t)} \mathbf{P}_{i j m}(t) e^{\curvearrowleft_{j}(t)-q_{j}(z)}
$$

Clearly, knowing the form of $\mathfrak{\Xi}(t)$, we can express the matrix $\mathbf{S}_{i j}(z, t)$ explicitly. In any case $\mathbf{S}_{i j}(z, t)$ is simply $\mathbf{P}_{i j m}(t)$ multiplied by a matrix polynomial in $\log (z / t)$. On substituting (3.14) into (3.13), integrating by parts and making use of (3.16), we find that

$\mathbf{R}_{i j m}^{*}(z)=\mathbf{U}_{i j m}^{*}(z)\left(z^{\eta_{1}-m}-\zeta^{\eta_{1}-m}\right)+\mathbf{V}_{i j m}^{1}(z)\left(z^{\eta_{1}-m-1}-\zeta^{\eta_{1}-m-1}\right)-\int_{\zeta}^{z} \mathbf{V}_{i j m}^{2}(z, t)\left(m+1-\eta_{1}\right) t^{-\left(m+2-\eta_{1}\right)} d t$,

where $\eta_{1}$ may be taken zero if $\mathbf{J}_{\mu_{i}}=\mathbf{O}$ for all $i=1,2, \ldots, l$, otherwise it is an arbitrary positive number less than 1 . The matrices $\mathbf{U}_{i j m}^{*}(z), \mathbf{V}_{i j m}^{1}(z)$ and $\mathbf{V}_{i j m}^{2}(z, t)$ are defined by

$$
\begin{gathered}
\mathbf{U}_{i j m}^{*}(z)=\frac{\mathbf{U}_{i j m} z^{-m} e^{\Sigma_{j}(z)-q_{j}(z)}-e^{\Sigma_{i}(z)-\varrho_{1}(\zeta)} \mathbf{U}_{i j m} \zeta^{-m} e^{\varrho_{j}(\zeta)-q_{j}(z)}}{z^{\eta_{1}-m}-\zeta^{\eta_{\Gamma}} m} \\
\mathbf{V}_{i j m}^{1}(z)=\frac{\mathbf{S}_{i j}(z, z) / q_{i j}^{\prime}(z)-e^{q_{i j}(z)-q_{i j}(\zeta)} \mathbf{S}_{i j}(z, \zeta) / q_{i j}^{\prime}(\zeta),}{z^{\eta_{1}-m-1}-\zeta^{\eta_{1}-m-1}}
\end{gathered}
$$


and

$$
\mathbf{V}_{i j m}^{2}(z, t)=\frac{t^{-\eta_{1}}}{m+1-\eta_{1}}\left[\frac{\partial}{\partial t}\left(\frac{\mathbf{S}_{i j}(z, t)}{q_{i j}^{\prime}(t)}\right) e^{q_{i j}(z)-q_{i j}(t)} t^{m+2}-e^{\Sigma_{k}(z)-\Sigma_{k}(t)} \sum_{k=1}^{m-1}\left\{\mathfrak{B}_{m-k}(t) \mathbf{U}_{j k}\right\}_{i} e^{\left.\mathfrak{D}_{j}(t)-q_{j}(z)\right)^{\prime}}\right],
$$

Equations (3.19) and (3.20) hold, provided that there is an integer $p$ in $0 \leqq p \leqq r$ such that $q_{i p}-q_{j p}$ $\neq 0$. If this is not so we define $\mathbf{V}_{i j m}^{1}(z)=0$, and

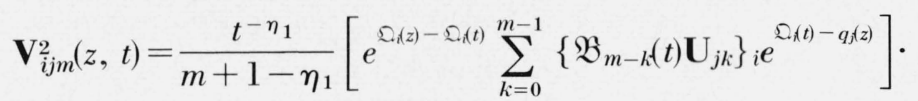

The representation (3.17) was motivated by the following consideration. We observe that each element of $\mathbf{R}_{i j m}^{*}(z)$ is of the form

$$
p(z, \zeta)=f(z)-f(\zeta)+g(z)-g(\zeta)+\int_{z}^{z} h(z, t) t^{-m-2} d t
$$

where $|f(z)|=\mathrm{O}\left(|z|^{-m}|\log z|^{\mu-\nu}\right)$ (see eq $\left.(2.27)\right), \quad|g(z)|=\mathrm{O}\left(|z|^{-m-1}|\log z|^{\mu-\nu}\right)$ and $h(z, t)=\mathrm{O}\left(|\log z|^{k}\right.$ $\left.|\log t|^{s}\right)$, as $|z|,|t| \rightarrow \infty$, where $k$ and $s$ are integers. The reason for introducing terms like $t^{-\eta}, t^{-\eta_{1}}$ where $\eta$ and $\eta_{1}$ are nonnegative numbers less than 1 is to eliminate logarithmic terms conveniently, and at the same time obtain good bounds. From (3.22), we have

$$
\begin{aligned}
|p(z, \zeta)| & \leqq\left|\frac{f(z)-f(\zeta)}{\left|z^{\eta_{1}-m}-\zeta^{\eta_{1}-m}\right|}\right| z^{\eta_{1}-m}-\zeta^{\eta_{1}-m} \mid \\
& +\left|\frac{g(z)-g(\zeta)}{z^{\eta_{1}-m-1}-\zeta^{\eta_{1}-m-1}}\left(z^{\eta_{1}-m-1}-\zeta^{\eta_{1}-m-1}\right)+\int_{\zeta}^{z} h(z, t) t^{-\eta_{1}} t^{\eta_{1}-m-2} d t\right| \\
& =\left|\frac{f(z)-f(\zeta)}{z^{\eta_{1}-m}-\zeta^{\eta_{1}-m} \mid}\right|\left(\eta_{1}-m\right) \int_{\zeta}^{z} t^{\eta_{1}-m-1} d t \mid \\
& +\left|\int_{\zeta}^{z}\left\{\frac{\left(\eta_{1}-m-1\right)(g(z)-g(\zeta))}{z^{\eta_{1}-m-1}-\zeta^{\eta_{1}-m-1}}+h(z, t) t^{-\eta_{1}}\right\} t^{\eta_{1}-m-2} d t\right|,
\end{aligned}
$$

from which

$|p(z, \zeta)| \leqq \sup _{t \in \mathscr{P}}\left|\frac{f(t)-f(\zeta)}{t^{\eta_{1}-m}-\zeta^{\eta_{1}-m}}\right| \mathscr{V}_{\mathscr{P}}\left(t^{\eta_{1}-m}\right)+\sup _{t, \tau \in \mathscr{P}}\left|\frac{g(\tau)-g(\zeta)}{\tau^{\eta_{1}-m-1}-\zeta^{\eta_{1}-m-1}}+\frac{h(\tau, t) t^{-\eta_{1}}}{\tau^{\eta_{1}-m-1}}\right| \mathscr{V}_{\mathscr{P}}\left(t^{\eta_{1}-m-1}\right)$

where in the last supremum $t$ and $\tau$ are points on $\mathscr{P}$ in order $\zeta, t, \tau, z$.

In each of the next two sections we first establish the existence and uniqueness of the solutions to the integral equations (3.8) and (3.11), then we obtain the error bounds. Our procedure is, in one aspect, similar to that in [6] in that we first establish that the conditions in sections 3.2 determine a domain. We then appeal to the contraction mapping principle (see e.g. [8], sec. 11) to establish existence and uniqueness. Proofs by use of successive approximations are given in [7].

\subsection{Error Bounds for the Extreme Eigenvalue Case}

We use the following notation to refer to the elements of a partitioned $n \times n$ matrix $\mathbf{A}$. $\mathbf{A}_{j}=\{\mathbf{A}\}_{j}$ will denote the $j$ th column of blocks in $\mathbf{A}, \mathbf{A}_{i j}=\left\{\mathbf{A}_{j}\right\}_{i}=\{\mathbf{A}\}_{i j}$ the $(i, j)$ th block, $\mathbf{A}_{i}^{\nu}=\left\{\mathbf{A}_{j}\right\}^{\nu}$ 
the $\nu$ th vector in the $j$ th column of blocks, and $A_{i j}^{\omega \nu}=\left\{A_{j}\right\}_{i}^{\omega \nu}=\left\{A_{j}^{\nu}\right\}_{i}^{\omega}$ the $(\omega, \nu)$ th element in the $(i, j)$ th block.

LEMMA 3.1: If under the extreme eigenvalue conditions of section $3.2, \mathscr{D}(\mathrm{z}, \zeta)$ contains at least one finite point $\mathrm{z}^{*}$, distinct from $\zeta$, then $\mathscr{D}(\mathrm{z}, \zeta)$ is a domain, that is, an open connected set of points.

Proof: The region $\mathscr{D}^{*}$ defined in Section 3.2 is certainly a domain. By assumption there is a path $\mathscr{P}$ in $\mathscr{D}^{*}$ connecting $z^{*}$ with $\zeta$ satisfying the three extreme eigenvalue conditions. Similarly there is such a path connecting an arbitrary finite point $z_{0}$ on $\mathscr{P}$ with $\zeta$. We can clearly choose a sufficiently small positive number $\rho$ such that the disk $\mathscr{S}(\rho):\left|z-z_{0}\right|<\rho$ lies entirely in $\mathscr{D}^{*}$, and when an arbitrary point $z_{1}$ of the disk is joined to $z_{0}$ by a straight line $\mathscr{P}_{1}$ then conditions (1) and (3) are satisfied on $\mathscr{P} \cup \mathscr{P}_{1}$. It remains to be shown that condition (2) is also satisfied.

To show this we define matrices

$$
\begin{gathered}
\mathbf{K}_{i}(\mathscr{P})=\sup _{\tau, t \in \mathscr{P}}\left|\exp \left[\mathfrak{D}_{i}(\tau)-\mathfrak{D}_{i}(t)-\mathbf{I}_{\mu_{i}}\left(q_{j}(\tau)-q_{j}(t)\right)\right]\right| \quad(i=1,2, . ., l ; i \neq j) \\
\left.K_{i}^{\prime}\left(\mathscr{P}_{1}\right)=\sup _{\tau_{1}, t_{1} \in \mathscr{P}_{1}} \mid \exp \left[\mathfrak{\beth}_{i}\left(\tau_{1}\right)-\mathfrak{D}_{i}\left(t_{1}\right)-\mathbf{I}_{\mu_{i}} q_{j}\left(\tau_{1}\right)-q_{j}\left(t_{1}\right)\right)\right] \mid,
\end{gathered}
$$

where $\tau, t, \tau_{1}, t_{1}$ are points on $\mathscr{P} \cup \mathscr{P}_{1}$ in the ordèr $z_{1}, \tau_{1}, t_{1}, z_{0}, \tau, t, \zeta$, and the suprema apply to individual elements of the matrices, as in [1]. Clearly, by hypothesis each of these matrices has nonnegative bounded elements. We now choose $\tau$ and $t$ on $\mathscr{P} \cup \mathscr{P}_{1}$ in the order $z_{1}, \tau, t, \zeta$. We then consider

$$
K_{i}\left(\mathscr{P} \cup \mathscr{P}_{1}\right)=\sup _{\tau, t \in \mathscr{P} \cup \mathscr{P}_{1}}\left|\exp \left[\bigcap_{i}(\tau)-\Im_{\mathfrak{l}_{i}}(t)-\mathbf{1}_{\mu_{i}}\left(q_{j}(\tau)-q_{j}(t)\right)\right]\right|
$$

where $i$ is a part of the range $(1, l)$ other than $j$. If $\tau$ is on $\mathscr{P}$ then so is $t$, and in this case by hypothesis the matrix on the right consists of nonnegative bounded elements. Similarly when $\tau$ and $t$ are on $\mathscr{P}_{1}$. Suppose then, that $\tau$ is on $\mathscr{P}_{1}$ and $t$ is on $\mathscr{P}$. In this case

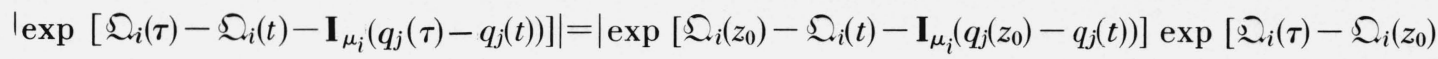

$\left.-\mathbf{I}_{\mu_{i}}\left(q_{j}(\tau)-q_{j}\left(z_{0}\right)\right)\right]|\leqq| \exp \left[\mathfrak{Q}_{i}\left(z_{0}\right)-\mathfrak{Q}_{i}(t)-\mathbf{I}_{\mu_{i}}\left(q_{j}\left(z_{0}\right)-q_{j}(t)\right)\right]|| \exp \left[\mathfrak{D}_{i}(\tau)-\mathfrak{S}_{i}\left(z_{0}\right)-\mathbf{I}_{\mu_{i}}\left(q_{j}(\tau)-q_{j}\left(z_{0}\right)\right)\right] \mid$,

so that $K_{i}\left(\mathscr{P} \cup \mathscr{P}_{1}\right) \leqq K_{i}(\mathscr{P}) K_{i}^{\prime}\left(\mathscr{P}_{1}\right)$. This shows that the elements of $K_{i}\left(\mathscr{P} \cup \mathscr{P}_{1}\right)(i=1,2, \ldots, l$; $i \neq j)$ are bounded, and therefore that $\mathscr{D}(z, \zeta)$ is a domain.

We next establish the existence and uniqueness of the solution of the integral equation (3.8). To this end, we observe that all known functions in (3.8) are holomorphic and single-valued in every simply-connected subset of $\mathscr{D}(z, \zeta)$. If $\mathscr{D}(\mathrm{z}, \zeta)$ is multiply-connected, we interpret $\mathscr{D}(\mathrm{z}, \zeta)$ to be a Riemann surface on which all known functions of equation (3.8) are holomorphic and single-valued.

Consider the set $\mathfrak{S}(\zeta)$ of all $n \times 1$ vectors $\mathbf{h}(z)$ of functions holomorphic in $\mathscr{D}(z, \zeta)$ such that $\lim _{z \rightarrow \zeta} \mathbf{h}(z)=\mathbf{O}$, where $z \rightarrow \zeta$ along some path $\mathscr{P}$ belonging to the family described above. The set 
$\mathfrak{S}(\zeta)$ together with the metric vector

$$
d\left(\mathbf{h}_{1}, \mathbf{h}_{2}\right)=\sup _{t \in \mathscr{P}}\left|\mathbf{h}_{1}(t)-\mathbf{h}_{2}(t)\right|
$$

forms a complete ${ }^{7}$ metric space.

Let

$$
\begin{aligned}
& \mathbf{U}_{j m}^{\nu *}=\sup _{\tau \in \mathscr{P}}\left|U_{j m}^{*}(\tau)\right| \\
& \boldsymbol{\gamma}_{j m}^{\nu}=\sup _{t, \tau \in \mathscr{P}}\left|\mathbf{V}_{j m}^{\nu 1}(\tau)+\mathbf{V}_{j m}^{\nu 2}(\tau, t)\right|
\end{aligned}
$$

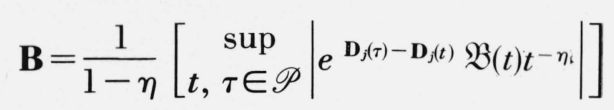

where for example $\mathbf{V}_{j m}^{\nu k}(k=1,2)$ is the $\nu$ th column vector in the column of blocks $\mathbf{V}_{j m}^{k}(k=1,2)$, and $t$ and $\tau$ are on $\mathscr{P}$ in the order $\zeta, t, \tau, z$. By the analysis of the previous section, all elements of the vectors and matrices defined in (3.28) to (3.30) are bounded. It follows that with $\epsilon_{j m}^{\nu}(t)$ replaced by $\mathbf{h}(t)$, the right of (3.8) is a contraction mapping of a complete metric space into itself, so that the solution to (3.8) exists and is unique.

On substituting the bounds (3.28) to (3.30) into (3.8) and using Lemma 2 of the appendix in [1], we obtain:

THEOREM 3.1: If, corresponding to a formal vector solution $\tilde{\mathbf{W}}_{\mathrm{jm}}^{\nu}(\mathbf{z})$ of equation (2.3), we can determine a region $\mathscr{D}(\mathrm{z}, \zeta)$ satisfying the extreme eigenvalue conditions of Section 3.2 , then equation (2.3) possesses an actual solution vector of functions holomorphic in $\mathscr{D}(\mathrm{z}, \zeta)$, given by

$$
\mathbf{W}_{\mathrm{jm}}^{\nu}(\mathrm{z})=\left[\sum_{\mathrm{k}=0}^{\mathrm{m}-1}\left(\sum_{\mathrm{s}=\nu}^{\mu} \mathbf{U}_{\mathrm{jk}}^{\mathrm{s}} \frac{(\log \mathrm{z})^{\mathrm{s}-\nu}}{(\mathrm{s}-\nu) !}\right) \mathrm{z}^{-\mathrm{k}}+\boldsymbol{\epsilon}_{\mathrm{jm}}^{\nu}(\mathrm{z})\right] \mathrm{e}^{q_{\mathrm{j}}(\mathrm{z})},
$$

in which

$$
\left|\boldsymbol{\epsilon}_{\mathrm{jm}}^{\nu}(\mathbf{z})\right| \leqq \exp \left\{\mathbf{B} \mathscr{V}_{\mathscr{P}}\left(\mathrm{t}^{\eta-1}\right)\right\}\left\{\mathbf{U}_{\mathrm{jm}}^{\nu * \mathscr{V}_{\mathscr{P}}}\left(\mathrm{t}^{\eta_{1}-\mathrm{m}}\right)+\gamma_{\mathrm{jm}}^{\nu} \mathscr{V}_{\mathscr{P}}\left(\mathrm{t}^{\eta_{1}-\mathrm{m}-1}\right)\right\}
$$

for all $\mathrm{z}$ in $\mathscr{D}(\mathrm{z}, \zeta)$, where the matrix $\mathbf{B}$ and the elements of the vectors $\mathbf{U}_{\mathbf{j} \mathrm{m}}^{\nu *}$ and $\boldsymbol{\gamma}_{\mathrm{jm}}^{\nu}$ are defined by equations (3.28) to (3.30). The solution $\mathbf{W}_{\mathrm{jm}}^{\nu}(\mathrm{z})$ depends on $\zeta$ and an arbitrary positive integer $\mathrm{m}$. The function $q_{j}(\mathrm{z})$ is defined in Theorem 2.1. The integers $\mu$ and $\nu$ and the vectors $\mathbf{U}_{\mathrm{jk}}^{\mathrm{s}}(\mathrm{k}=0$, $1,2, \ldots ; \mathrm{s}=1,2, \ldots, \mu)$ are defined as for equation $(2.27) . \quad$ If each $\mathbf{J}_{\mu_{\mathrm{j}}}=\mathrm{O}(\mathrm{i}=1,2, \ldots, l)$ then the numbers $\eta$ and $\eta_{1}$ can be taken to be zero; if $\mathbf{J}_{\mu_{\mathrm{j}}}=\mathbf{O}$ then $\eta$ can be taken to be zero; otherwise $\eta$ and $\eta_{1}$ are arbitrary positive numbers less than 1.

Let us now obtain a norm bound for the vector $\epsilon_{j m}^{\nu}(z)$ in Theorem 3.1. To this end we use the definitions (3.18) to (3.21), and define

$$
\begin{gathered}
B=\frac{1}{1-\eta} t, \quad \sup _{\tau \in \mathscr{P}}\left\|e^{\mathbf{D}_{j}(\tau)-\mathbf{D}_{j}(t)} \mathfrak{B}(t) t^{-\eta}\right\|, \\
\mathbf{V}_{j m}^{\nu *}=\sup _{\tau \in \mathscr{P}}\left\|\mathbf{U}_{j m}^{\nu *}(\tau)\right\|, \quad \gamma_{j m}^{\nu}=\sup _{t, \tau \in \mathscr{P}}\left\|\mathbf{V}_{j m}^{\nu 1}(\tau)+\mathbf{V}_{j m}^{\nu 2}(\tau, t)\right\|,
\end{gathered}
$$

${ }^{7}$ This is easily verified by use of Vitali's theorem. 
where $t$ and $\tau$ are on $\mathscr{P}$ in the order $\zeta, t, \tau, z$. Substituting these quantities into (3.8) and using [1] Lemma 2, appendix, we obtain

THEOREM 3.2: A norm bound for the vector $\boldsymbol{\epsilon}_{\mathrm{jm}}^{\nu}(\mathrm{z})$ in Theorem 3.1 is given by

$$
\left\|\boldsymbol{\epsilon}_{\mathrm{jm}}^{\nu}(\mathrm{z})\right\| \leqq \exp \left\{B \mathscr{V}_{\mathscr{P}}\left(\mathrm{t}^{\eta-1}\right)\right\}\left\{U_{\mathrm{jm},}^{\nu *} \mathscr{V}_{\mathcal{P}}\left(\mathrm{t}^{\eta_{1}-\mathrm{m}}\right)+\gamma_{\mathrm{jm}}^{\nu} \mathscr{V}_{\mathscr{P}}\left(\mathrm{t}^{\eta_{1}-\mathrm{m}-1}\right)\right\}
$$

where $\eta$ and $\eta_{1}$ are defined as in Theorem 3.1 and $\mathbf{B}, \mathbf{U}_{\mathrm{jm}}^{\nu *}$ and $\gamma_{\mathrm{jm}}^{\nu}$ are defined in (3.33) and (3.34).

\subsection{Error Bounds for the Interior Eigenvalue Case}

We shall first establish the existence and uniqueness of the solution of (3.11) by use of norms. We split the vectors $\mathbf{U}_{j m}^{\nu *}(z), \mathbf{V}_{j m}^{\nu k}(k=1,2)$ defined in (3.18) in the manner corresponding to the way (3.7) was split in (3.10) into lower dimensional vectors $\mathbf{U}_{j m s}^{\nu *}(z), \mathbf{V}_{j m s}^{\nu k}(s=1,2)$. We change $\zeta$ to $\zeta_{s}$ if $i \in \mathbf{N}_{s}(s=1,2:$ in the notation of the interior eigenvalue conditions). We then define

$$
\begin{aligned}
\psi_{m k}(u, v)=\sup _{t_{2} \in \mathscr{P}}\left\|\mathbf{V}_{j m k}^{\nu 1}\left(t_{2}\right)\right\| \mathscr{V}_{u, v}\left(t^{\eta_{1}-m-1}\right) & \\
& +\sup _{t_{1}, t_{2} \in \mathscr{P}}\left\{\left\|\mathbf{V}_{j m k}^{\nu 1}\left(t_{2}\right)+\mathbf{V}_{j m k}^{\nu 2}\left(t_{2}, t_{1}\right)\right\|\right\} \mathscr{V}_{u, v}\left(t^{\eta_{1}-m-1}\right)(k=1,2)
\end{aligned}
$$

where $t_{1}$ and $t_{2}$ are on $\mathscr{P}$ in the order $\zeta_{1}, t_{1}, t_{2}, \zeta_{2}$ if $k=1$, or $\zeta_{1}, t_{2}, t_{1}, \zeta_{2}$ if $k=2$. The fixed number $\eta_{1}$ may be taken to be zero if $\mathbf{J}_{\mu_{i}}=\mathbf{O}(i=1,2, \ldots, l)$ but $\eta_{1}$ is otherwise an arbitrary positive number less than 1 .

Similarly, we define

$$
a_{k}=\sup _{t_{1}, t_{2} \in \mathscr{P}}\left\|\frac{t_{2}^{-\eta}}{1-\eta} e^{\mathbf{D}_{j s}\left(t_{2}\right)-\mathbf{D}_{j s}\left(t_{1}\right)} \mathfrak{a}_{k}\left(t_{1}\right)\right\| \quad\left\{\begin{array}{l}
s=1, k=1,2 \\
s=2, k=3,4
\end{array}\right.
$$

where $t_{1}$ and $t_{2}$ are again on $\mathscr{P}$ in the order $\zeta_{1}, t_{1}, t_{2}, \zeta_{2}$ if $s=1, k=1,2$ and in the order $\zeta_{1}, t_{2}, t_{1}, \zeta_{2}$ if $s=2, k=3,4$. The number $\eta$ may be taken to be zero if $\mathbf{J}_{\mu_{j}}=\mathbf{O}$ but otherwise $\eta$ is an arbitrary positive number less than 1.

LEMMA 3.2: If under the interior eigenvalue conditions of section $3.2, \mathscr{D}\left(\zeta_{1}, \zeta_{2}, \eta\right)$ contains at least one finite point $\mathrm{z}^{*}$ distinct from $\zeta_{1}$ and $\zeta_{2}$, then $\mathscr{D}\left(\zeta_{1}, \zeta_{2}, \eta\right)$ is a domain.

Proof: By assumption there is a path $\mathscr{P}$ in $\mathscr{D}^{*}\left(\mathscr{D}^{*}\right.$ defined in sec. 3.2$)$ connecting $\zeta_{1}, z^{*}$ and $\zeta_{2}$ (in that order) and satisfying the three interior eigenvalue conditions. The same path $\mathscr{P}$ con-

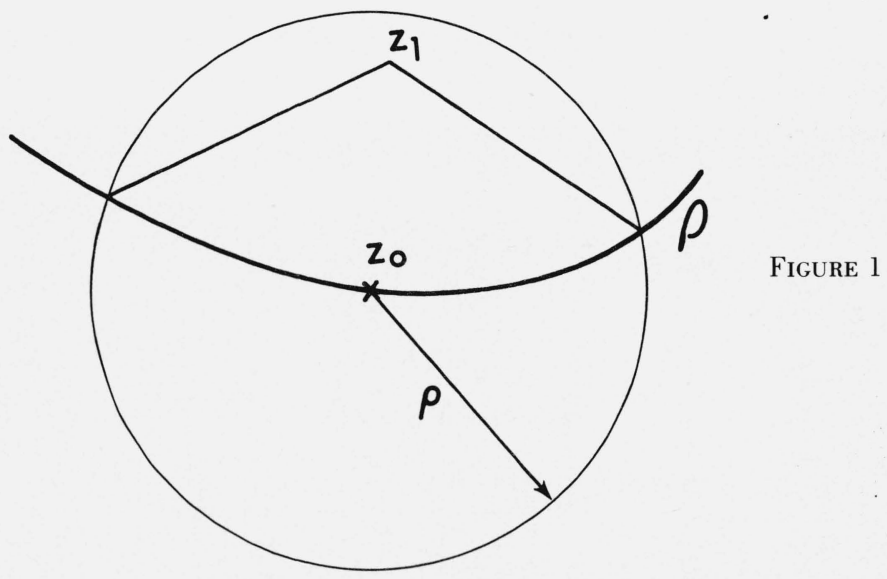


nects an arbitrary point $z_{0}$ on $\mathscr{P}$ in this manner. By an argument similar to that used in the proof of Lemma 3.1, we can clearly choose a sufficiently small number $\rho>0$ such that when we alter $\mathscr{P}$ to pass through an arbitrary point $z_{1}$ in the interior of the disk $\mathscr{S}(\rho)$ instead then (1) and (3) of the interior eigenvalue conditions remain satisfied. To prove that condition (2) also remains satisfied we need merely show that if $\rho \rightarrow 0$ and the altered path $\mathscr{P}^{\prime}$ in figure 1 tends to $\mathscr{P}$, then

$$
\sup _{t, \tau \in \mathscr{P}^{\prime}}\left\|e^{\mathbf{D}_{j_{1}(\tau)-\mathbf{D}_{j i}(t)}} a_{1}(t) t^{-\eta}\right\| \rightarrow \sup _{t, \tau \in \mathscr{P}}\left\|e^{\mathbf{D}_{j(1}(\tau)-\mathbf{D}_{j_{1}(t)}} a_{1}(t) t^{-\eta}\right\|
$$

where $t$ and $\tau$ are points located on the paths $\mathscr{P}$ and $\mathscr{P}^{\prime}$ in the order $\zeta_{1}, t, \tau, \zeta_{2}$. A convenient way of achieving this is to put

$$
\Delta\left(t, t_{1}, \tau, \tau_{1}\right)=e^{\mathbf{V}_{j_{1}}\left(\tau_{1}\right)-\mathbf{D}_{j_{2}}\left(t_{1}\right)} \mathfrak{a}_{1}\left(t_{1}\right) t_{1}^{-\eta}-e^{\mathbf{D}_{j_{1}}(\tau)-\mathbf{D}_{j_{1}}(t)} a_{1}(t) t^{-\eta}
$$

where $\tau$ and $t$ are located as described above; outside $\mathscr{S}(\rho), t_{1}=t, \tau_{1}=\tau$ while in the interior of $\mathscr{S}(\rho) t_{1}$ and $\tau_{1}$ are arbitrarily located on $\mathscr{P}^{\prime}$. Let us denote the norm on the left of (3.38) by $\alpha^{\prime}$, that on the right by $\alpha$. Then it follows that

$$
\alpha^{\prime}=\alpha+\sup \left\|\mathbf{\Delta}\left(t, t_{1}, \tau, \tau_{1}\right)\right\|,
$$

where the supremum is taken for $t_{1}, \tau_{1} \in \mathscr{P}^{\prime}$ and $t, \tau \in \mathscr{P}$. The norm on the right is clearly a continuous function of its elements, and tends to zero as $\rho \rightarrow 0$. A similar argument applies to $a_{2}$, $\mathfrak{a}_{3}$ and $\mathfrak{a}_{4}$. Moreover the difference $\mathscr{V}_{\mathscr{P}}\left(t^{\eta-1}\right)-\mathscr{V}_{\mathscr{\Phi}}^{\prime}\left(\eta^{\eta-1}\right)$ can be made as small as we please by taking $\rho$ sufficiently small. The truth of Lemma 3.2 is now evident.

We again observe that if $\mathscr{D}\left(\zeta_{1}, \zeta_{2}, \eta\right)$ is simply connected then all known functions appearing in equation (3.11) are single valued in this domain. If, on the other hand, $\mathscr{D}\left(\zeta_{1}, \zeta_{2}, \eta\right)$ is multiply connected then we interpret $\mathscr{D}\left(\zeta_{1}, \zeta_{2}, \eta\right)$ as a Riemann surface on which all known functions appearing in eq. (3.11) are single valued.

Consider the $\operatorname{set}^{8} \mathfrak{S}_{\mathrm{e}}\left(\zeta_{1}, \zeta_{2}\right)=\left(\mathfrak{S}_{1}\left(\zeta_{1}, \zeta_{2}\right), \mathfrak{S}_{2}\left(\zeta_{1}, \zeta_{2}\right)\right)^{T}$ of $n \times 1$ column vectors $\mathbf{h}(z)=\left(\mathbf{h}_{1}(z), \mathbf{h}_{2}(z)\right)^{T}$ of functions holomorphic in $\mathscr{D}\left(\zeta_{1}, \zeta_{2}, \eta\right)$, such that $\mathbf{h}_{1}(z) \in \mathfrak{S}_{1}\left(\zeta_{1}, \zeta_{2}\right)$ is $\kappa \times 1, \mathbf{h}_{2}(z) \in \mathfrak{S}_{2}\left(\zeta_{1}, \zeta_{2}\right)$ is $(n-\kappa) \times 1$, and all elements of $\mathbf{h}(z)$ are bounded as $z \rightarrow \zeta_{1}, z \rightarrow \zeta_{2}$ along any path $\mathscr{P}$ of the family described above. If we take

$$
\begin{aligned}
& \sup _{t \in \mathscr{P}}\left\|\mathbf{x}_{1}(t)-\mathbf{y}_{1}(t)\right\| \\
& d(x, y)= \\
& \left(\sup _{t \in \mathscr{P}}\left\|\mathbf{x}_{2}(t)-\mathbf{y}_{2}(t)\right\|\right)
\end{aligned}
$$

as metric for $\mathfrak{H}_{\mathcal{C}}$, where $\mathbf{x}_{k}(t), \mathbf{y}_{k}(t) \in \mathfrak{S}_{k}\left(\zeta_{1}, \zeta_{2}\right)(k=1,2)$, then it follows that $\mathfrak{S}_{\mathfrak{C}}\left(\zeta_{1}, \zeta_{2}\right)$ together with the metric (3.41)i form a complete metric space. Substituting (3.36) and (3.37) in (3.11) and using the fact that the eigenvalue of largest modulus of the matrix $\left(\begin{array}{ll}a_{1}, & a_{3} \\ a_{2}, & a_{4}\end{array}\right) \mathscr{V}_{\mathscr{P}}\left(t^{\eta-1}\right)$ is $\frac{1}{2}\left\{a_{1}+a_{4}\right.$ $\left.+\sqrt{\left(a_{1}-a_{4}\right)^{2}+4 a_{2} a_{3}}\right\} \mathscr{q}_{\mathscr{P}}\left(t^{\eta-1}\right)$ and therefore less than 1 , we see that the right of (3.11) is a contraction mapping of a complete metric space into itself. Hence the solution of (3.11) exists and is unique.

Following [1] we shall again first obtain a vector bound. We thus define $\psi_{m k}(u, v)$ and $\mathbf{a}_{k}$ as in (3.36) and (3.37) respectively, except that the norms in these equations are now replaced by absolute values. Thus $\psi_{m k}$ and $\mathbf{a}_{k}$ are now matrices of absolute values. On substituting these bounds into (3.11) and proceeding as in [1] we obtain

${ }^{8}$ The index $T$ denotes matrix transposition. 
THEOREM 3.3: If, corresponding to the vector $\tilde{\mathbf{W}}_{j m}^{\nu}(z)(c f$. , eq. (2.26)) we can determine a region $\mathscr{D}\left(\zeta_{1}, \zeta_{2}, \eta\right)$ satisfying the interior eigenvalue conditions of section 3.2 , then the eq. (2.3) possesses an actual solution vector of functions holomorphic in $\mathscr{D}\left(\zeta_{1}, \zeta_{2}, \eta\right)$ and depending on $\mathrm{m}, \zeta_{1}$ and $\zeta_{2}$, given by

$$
\left(\begin{array}{l}
\mathbf{W}_{\mathrm{j} \mathrm{m} 1}^{\nu}(\mathrm{z}) \\
\mathbf{W}_{\mathrm{j} \mathrm{m} 2}^{\nu}(\mathrm{z})
\end{array}\right)=\left[\sum_{\mathrm{k}=0}^{\mathrm{m}-1}\left\{\sum_{\mathrm{s}=\nu}^{\mu}\left(\begin{array}{c}
\mathrm{U}_{\mathrm{jk} 1}^{\mathrm{s}} \\
\mathrm{U}_{\mathrm{jk} 2}^{\mathrm{s}}
\end{array}\right) \frac{(\log \mathrm{z})^{\mathrm{s}-\nu}}{(\mathrm{s}-\nu) !}\right\} \mathrm{z}^{-\mathrm{k}}+\left(\begin{array}{c}
\boldsymbol{\epsilon}_{\mathrm{j} \mathrm{m} 1}^{\nu}(\mathrm{z}) \\
\boldsymbol{\epsilon}_{\mathrm{j} \mathrm{m} 2}^{\nu}(\mathrm{z})
\end{array}\right)\right] \mathrm{e}^{q_{\mathrm{j}}(\mathrm{z})}
$$

Here

$$
\left|\boldsymbol{\epsilon}_{\mathrm{jm} 1}^{\nu}(\mathbf{z})\right| \leqq \exp \left\{\mathbf{a}_{2} \mathscr{V}_{\zeta_{1}, \mathbf{z}}\left(\mathrm{t}^{\eta-1}\right)\right\} \psi_{\mathrm{m} 1}\left(\zeta_{1}, \mathrm{z}\right)+\mathbf{C}_{1}\left(\zeta_{1}, \mathrm{z}\right)\left[\exp \left\{\mathbf{a}_{4} \mathscr{V}_{\mathcal{P}}\left(\mathbf{t}^{\eta-1}\right)\right\} \psi_{\mathrm{m} 2}\left(\zeta_{1}, \zeta_{2}\right)+\mathbf{B}\right]
$$

and $\mathbf{a}_{1}, \mathbf{a}_{2}, \mathbf{a}_{3}$, and $\mathbf{a}_{4}$ are defined by (3.37), $\Psi_{\mathrm{mk}}(\mathrm{u}, \mathrm{v})(\mathrm{k}=1,2)$ are defined by (3.36) with norms in these equations replaced by absolute values, and

$$
\mathbf{B}=\left[\mathbf{I}_{2}-\mathbf{C}_{2} \mathbf{C}_{1}\left(\zeta_{1}, \zeta_{2}\right)\right]^{-1} \mathbf{C}_{2} \times \exp \left\{\mathbf{a}_{1} \mathscr{V}_{\mathcal{P}}\left(t^{\eta-1}\right)\right\} \Psi_{\mathrm{m} 1}\left(\zeta_{1}, \zeta_{2}\right)+\mathbf{C}_{1}\left(\zeta_{1}, \zeta_{2}\right) \exp \left\{\mathbf{a}_{4} \mathscr{V}_{\mathscr{P}}\left(t^{\eta-1}\right)\right\} \Psi_{\mathrm{m} 2}\left(\zeta_{1}, \zeta_{2}\right)
$$

$$
\mathbf{C}_{1}\left(\zeta_{1}, \mathbf{z}\right)=\mathbf{a}_{1}^{-1}\left[\exp \left\{\mathbf{a}_{1} \mathscr{V}_{\zeta_{1}}, \zeta\left(t^{\eta-1}\right)\right\}-\mathbf{I}_{1}\right] \mathbf{a}_{2} ; \mathbf{C}_{2}=\mathbf{a}_{4}^{-1}\left[\exp \left\{\mathbf{a}_{4} \mathscr{V}_{\mathscr{P}}\left(\mathbf{t}^{\eta-1}\right)\right\}-\mathbf{I}_{2}\right]
$$

In (3.44) $\mathbf{I}_{1}$ designates a $\kappa \times \kappa$ unit matrix and $\mathbf{I}_{2}$ designates an $(n-\kappa) \times(n-\kappa)$ unit matrix, $\kappa$ being the number of elements in $\mathbf{N}_{1}$ (sec. 3.2). All variations on the right of (3.43), (3.44), and (3.45) are taken along $\mathscr{P}$. The function $q_{j}(\mathrm{z})$ is defined as in Theorem 2.1. The vector $\mathbf{U}_{\mathrm{jk}}^{\mathrm{s}}$ given by Theorem 3.1 is partitioned into lower dimensional vectors as described in section 3.2. The numbers $\eta$ and $\eta_{1}$ are defined as in Theorem 3.1. The bound (3.43) is valid when every eigenvalue of the matrix $\mathbf{C}_{2} \mathbf{C}_{1}\left(\zeta_{1}, \zeta_{2}\right)$ is less than 1 in magnitude. An exactly similar result holds for $\epsilon_{\mathrm{jm} 2}^{\nu}(\mathrm{z})$. We also have

Theorem 3.4: Let $\mathrm{a}_{1}, \mathrm{a}_{2}, \mathrm{a}_{3}$, and $\mathrm{a}_{4}$ be defined by (3.37), $\Psi_{\mathrm{mk}}(\mathrm{k}=1,2)$ be defined by (3.36), and

$$
\begin{aligned}
& f(z)=\mathscr{V}_{\zeta_{1}, z}\left(t^{\eta-1}\right), \\
& \mathrm{F}(\mathrm{z})=\frac{\mathrm{a}_{1} \mathrm{a}_{3}}{\left(\mathrm{a}_{1}+\mathrm{a}_{1}\right)^{2}}\left[\mathrm{e}^{\left(\mathrm{a}_{1}+\mathrm{a}_{4}\right) \mathrm{f}(\mathrm{z})}-1-\left(\mathrm{a}_{1}+\mathrm{a}_{4}\right) \mathrm{f}(\mathrm{z})\right],
\end{aligned}
$$

where the variations are taken along $\mathscr{P}$. If $\mathrm{F}\left(\zeta_{2}\right)<1$ then $1-\mathrm{F}\left(\zeta_{2}\right)$ times the norm of the vector $\boldsymbol{\epsilon}_{\mathrm{jml}}^{\nu}(\mathrm{z})(c f$. eq. (3.42)) is bounded by the expression

$\mathrm{e}^{\mathrm{a}_{1} \mathrm{f}(\mathrm{z})}\left[\mathrm{F}(\mathrm{z})+1-\mathrm{F}\left(\zeta_{2}\right)\right] \Psi_{\mathrm{m} 1}\left(\zeta_{1}, \mathrm{z}\right)$

$$
\begin{aligned}
+\frac{\mathrm{a}_{2} \mathrm{a}_{3}}{\left(\mathrm{a}_{1}+\mathrm{a}_{4}\right)^{2}}\left[1-\mathrm{e}^{-\left(\mathrm{a}_{1}+\mathrm{a}_{4}\right) \mathrm{f}(\mathrm{z})}\right]\left[\mathrm{e}^{\left(\mathrm{a}_{2}+\mathrm{a}_{4}\right) \mathrm{f}\left(\zeta_{2}\right)}-\mathrm{e}^{\left(\mathrm{a}_{1}+\mathrm{a}_{4}\right) \mathrm{f}(\mathrm{z})}\right] \Psi_{\mathrm{m} 1}\left(\mathrm{z}, \zeta_{2}\right) \\
+\frac{\mathrm{a}_{2}}{\mathrm{a}_{1}+\mathrm{a}_{4}} \mathrm{e}^{\mathrm{a}_{1} \mathrm{f}(\mathrm{z})+\mathrm{a}_{4} \mathrm{f}\left(\zeta_{2}\right)}\left[1-\mathrm{e}^{-\left(\mathrm{a}_{1}+\mathrm{a}_{4}\right) \mathrm{f}(\mathrm{z})}\right] \Psi_{\mathrm{m} 2}\left(\zeta_{1}, \zeta_{2}\right)
\end{aligned}
$$

An exactly similar result holds for $\boldsymbol{\epsilon}_{\mathrm{j} 2}^{\nu}(\mathbf{z})$. 


\section{Example: The Case $n=2$}

In this section we consider the special system $\mathbf{X}^{\prime}=z^{h \mathfrak{U}(z) \mathbf{X}}(h$ an integer), that is

$$
\left(\begin{array}{cc}
x_{11}^{\prime} & x_{12}^{\prime} \\
x_{21}^{\prime} & x_{22}^{\prime}
\end{array}\right)=z^{h}\left(\begin{array}{ll}
a_{11}(z) & a_{12}(z) \\
a_{21}(z) & a_{22}(z)
\end{array}\right)\left(\begin{array}{cc}
x_{11} & x_{12} \\
x_{21} & x_{22}
\end{array}\right)
$$

where for each arbitrary positive integer $s$

$$
a_{i j}(z)=\sum_{k=0}^{s-1} a_{i j k} z^{-k}+a_{i j s}(z) z^{-s} \quad(i, j=1,2)
$$

and the functions $a_{i j s}(z)(s=0,1,2, \ldots)$ are holomorphic in a domain $\mathscr{D}^{\prime}$ and uniformly bounded on some path $\mathscr{P} \in \mathscr{D}^{\prime}$ extending to infinity.

We begin by transforming the system (4.1) to canonical form. The flow chart on the following pages indicates the steps involved in transforming the system (4.1) to canonical form. The chart is constructed along the lines of the proof of Turrittin's theorem [3], and it contains all the transformations used in [3] for arbitrary $n$. The process terminates in all cases.

Box \#2 in the chart contains the normalizing transformation, box \#8 the exponential transformation, box \#19 the shearing transformation, box \#27 the zero-inducing transformation, and box \#31 the root-equalizing transformation. Box \#7 also contains a modified form of the zeroinducing transformation.

The resulting system takes the form

$$
\frac{d \mathbf{W}}{d z}=z^{r} \subseteq(z) \mathbf{W}=\left[\mathfrak{Q}^{\prime}(z)+z^{-2} \mathfrak{B}(z)\right] \mathbf{W}
$$

in which $r$ is an integer, and

$$
z^{r} \subseteq(z)=\left\{\left(\begin{array}{ll}
q_{1}^{\prime}(z) & 0 \\
0 & q_{2}^{\prime}(z)
\end{array}\right)+\left(\begin{array}{ll}
0 & 0 \\
\xi z^{-1} & 0
\end{array}\right)\right\}+z^{-2}\left\{\left(\begin{array}{ll}
b_{11}(z) & b_{12}(z) \\
b_{21}(z) & b_{22}(z)
\end{array}\right)\right\}
$$

$\mathfrak{Z}^{\prime}(z)$ is the first matrix in braces on the right, $\mathfrak{B}(z)$ is the second, and

$$
q_{i}(z)=\sum_{k=0}^{r} \frac{q_{i k}}{r+1-k} z^{r+1-k}+q_{i, r+1} \log z \quad(i=1,2) .
$$

If $r \leqq-2$ then $\mathfrak{Q}^{\prime}(z)=0$. If for some integer $p$ in the range $0 \leqq p \leqq r+1$, we have $q_{1 p} \neq q_{2 p}$, then $\xi=0$. Otherwise $\xi$ is either 0 or 1 . The functions $b_{i j}(z)(i=1,2)$ have the expansion

$$
b_{i j}(z)=\sum_{k=0}^{s-1} b_{i j k} z^{-k}+b_{i j s}(z) z^{-s},
$$

where the elements of $b_{i j s}(z)$ are holomorphic and uniformly bounded on a path $\mathscr{P}$ extending to infinity in $\mathscr{D}$.

By the analysis of sections 2 to 4 , the system (4.3) has a formal solution matrix of the form

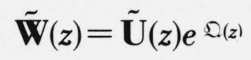


FLOW CHART ILLUSTRATING TRANSFORMATION OF THE SYSTEM TO CANONICAL FORM WHEN $n=2$

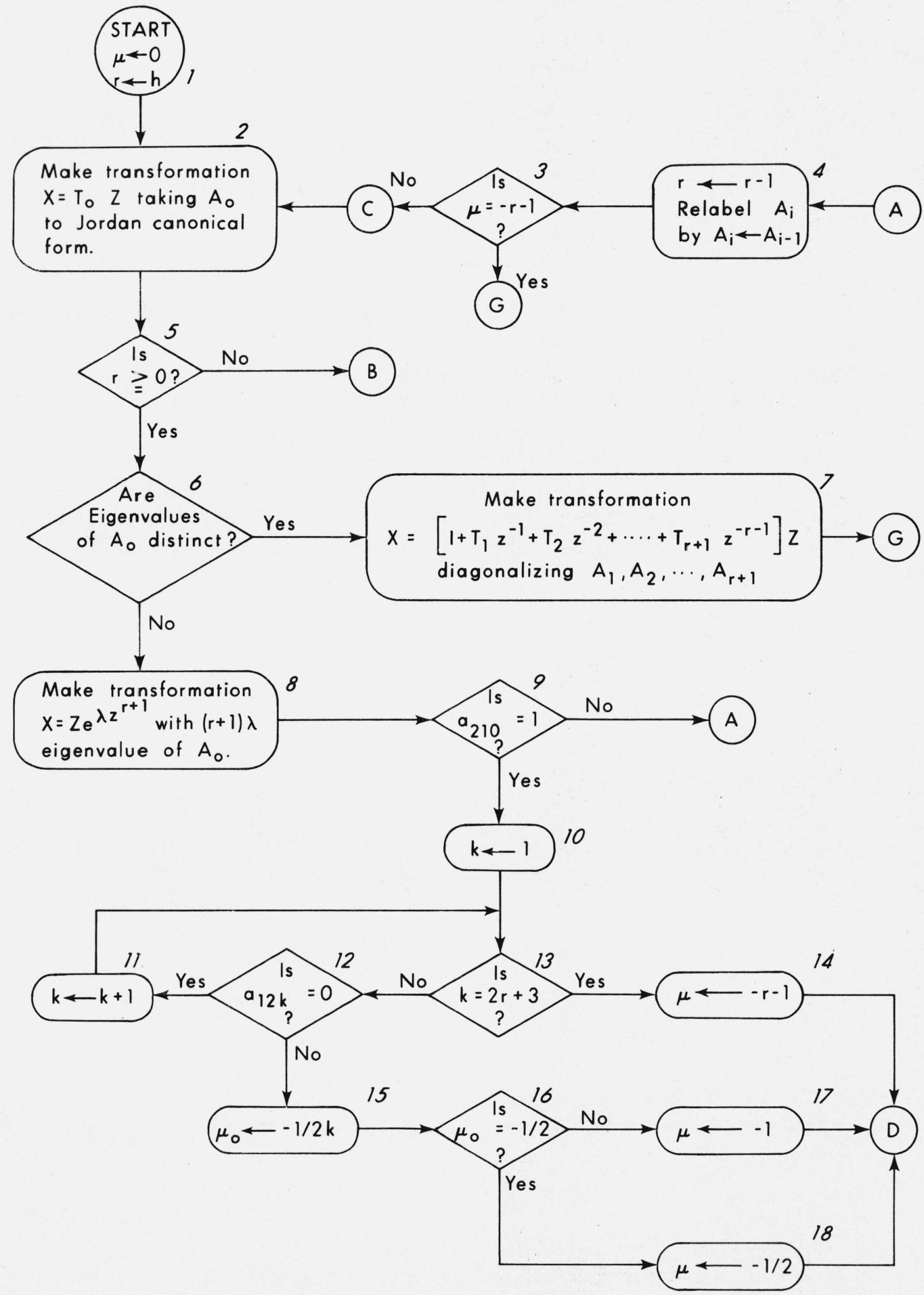

Figure 2 
21

Make transformations

$z=\zeta^{2}, \zeta=z$

$r \longleftarrow 2 r$

Redefine $a_{i j k}$ 's by

$b_{i j, 2 k+1} \longleftarrow 0(i \neq j)$;

$b_{i i, 2 k} \longleftarrow 0$;

$b_{12,2 k} \longleftarrow 2 a_{12, k+1}$

$b_{21,2 k} \longleftarrow 2 a_{21, k}$

$\mathrm{b}_{\mathrm{ii}, 2 \mathrm{k}+1} \longleftarrow 2 \mathrm{a}_{\mathrm{ii}, \mathrm{k}+1}$

$(k=0,1,2, \cdots)$

$b_{11,2 r+1} \longleftarrow 1+a_{11, r+1}$

$a_{i j k} \longleftarrow b_{i j k}$ $(k=0,1,2, \cdots)$
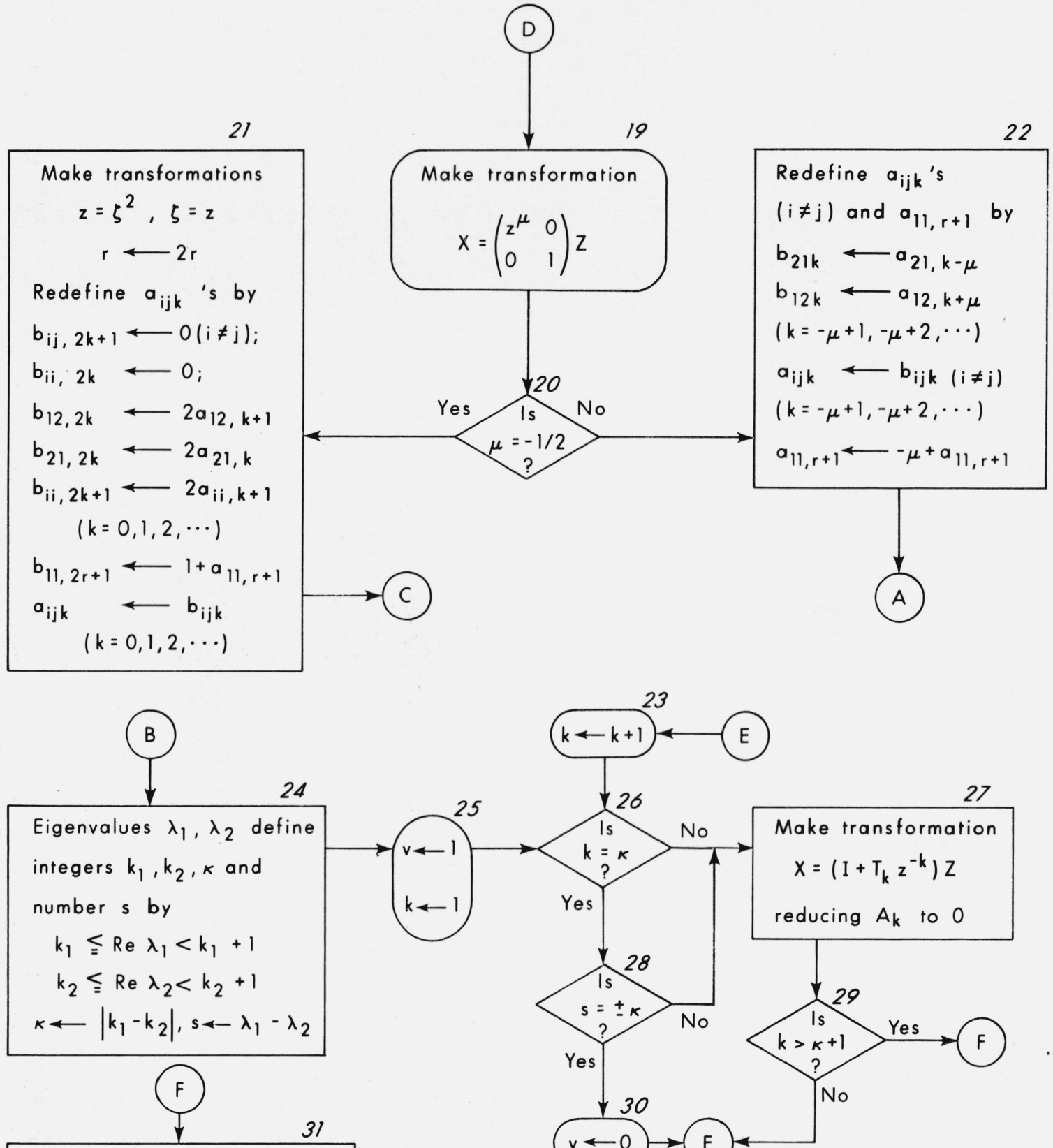

23

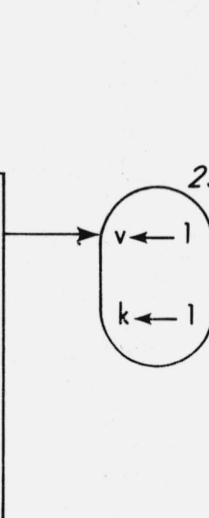

$25 \lcm{26}$

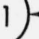

(E)

If solution corresponding to

$\lambda_{1}$ is desired, make transformation.

$$
x=\left(\begin{array}{cc}
z^{k_{1}+v} & 0 \\
0 & z^{k_{2}}
\end{array}\right) z
$$

Otherwise

$$
x=\left(\begin{array}{cc}
z^{k_{1}} & 0 \\
0 & z^{k_{2}+v}
\end{array}\right) z
$$

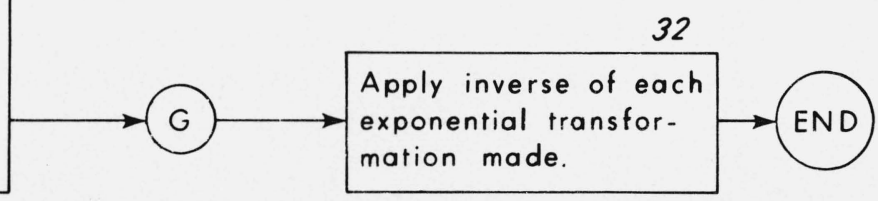

Figure 2-Continued 
where

$$
\begin{gathered}
\Im(z)=\left(\begin{array}{ll}
q_{1}(z) & 0 \\
0 & q_{2}(z)
\end{array}\right)+\left(\begin{array}{ll}
0 & 0 \\
\xi \log z & 0
\end{array}\right) \\
\widetilde{\mathbf{U}}(z) \triangleq \sum_{k=0}^{\infty} \mathbf{U}_{k} z^{-k} ; \quad \mathbf{U}_{0}=\mathbf{I} \\
\mathbf{U}_{k}=\left(\begin{array}{ll}
u_{11 k} & u_{12 k} \\
u_{21 k} & u_{22 k}
\end{array}\right) k=0,1,2, \ldots .
\end{gathered}
$$

Suppose that $p$ is the smallest integer in the range $0 \leqq p \leqq r+1$ such that $q_{1 p} \neq q_{2 p}$. Then the $u_{i j k}$ are computed from ${ }^{9}$

$$
\begin{aligned}
u_{i i 0} & =1 \quad(i=1,2) \\
u_{i j k} & =0 \quad \text { if } i \neq j \text { and } 0 \leqq k \leqq r+1-p \quad(i, j=1,2) \\
u_{i i k} & =-\frac{1}{k} \sum_{s=0}^{k-1}\left(b_{i 1 s} u_{1 i, k-1-s}+b_{i 2 s} u_{2 i, k-1-s}\right) \quad(i=1,2 ; k \geqq 1) \\
u_{i j, r+2+k-p} & =\frac{1}{q_{i p}-q_{j p}} \sum_{s=p+1}^{r+1} u_{i j, r+2+k-s}\left(q_{j s}-q_{i s}\right)+(k+1) u_{i j, k+1} \\
& +\sum_{s=0}^{k}\left(b_{i 1 s} u_{i j, k-s}+b_{i 2 s} u_{2 j, k-s}\right) \quad(i \neq j ; \quad k \geqq 0) .
\end{aligned}
$$

If, on the other hand, $q_{1 p}-q_{2 p}=0$ for $p=0,1, \ldots, r+1$, then $\mathbf{U}_{k+1}\left(k \geqq 0 ; \mathbf{U}_{0}=\mathbf{I}\right)$ is computed from

$$
(k+1) \mathbf{U}_{k+1}+\mathbf{J} \mathbf{U}_{k+1}-\mathbf{U}_{k+1} \mathbf{J}+\sum_{s=0}^{k} \mathbf{B}_{s} \mathbf{U}_{k-s}=\mathbf{O}
$$

with

$$
\mathbf{J}=\left(\begin{array}{ll}
0 & 0 \\
\xi & 0
\end{array}\right) ; \quad \xi=0 \text { or } 1
$$

In either case, whether $\xi=0$ or 1 , eq (4.10) with $\mathbf{U}_{0}=\mathbf{I}$ uniquely determines each $\mathbf{U}_{k}, k \geqq 1$. If $\xi=0$ each column vector can be computed independently of the other. If $\xi=1$ the second column vector in $\mathbf{U}_{k}(k=0,1, \ldots)$ can be computed without computing the first. If, on the other hand, $\xi=1$ and only the first column vector in $\mathbf{U}_{k}(k=0,1,2 \ldots)$ is required, the second column vector must also be computed, and the computations for the second column vector must precede the computations for the first.

We shall obtain a bound on the difference between a partial sum of the first formal solution vector and a corresponding actual solution vector. Since the analysis is similar to the preceding extreme eigenvalue case, we only present the main points.

${ }^{9}$ The sum $\sum_{s=p+1}^{r+1}$ is to be replaced by zero if $p=r+1$ 
In order to define a domain, we choose a nonnegative number $a$ such that if $q_{1 p}=q_{2 p}$ for $p=0,1, \ldots, r$, then $a=0$ if $|\zeta|=\infty$ and $a=|\zeta|$ if $|\zeta|<\infty$. Let us denote the largest zero of $q_{21}^{\prime}(z)$ by $a_{0}$. If for some $p \in(0,1, \ldots, r) q_{1 p} \neq q_{2 p}$ then we set $a=\left|a_{0}\right|$ if $|\zeta|=\infty$, while if $|\zeta|<\infty$ we assume that $\zeta \in \mathscr{D}, \zeta$ does not coincide with a zero of $q_{21}^{\prime}(z)$ and $|\zeta| \geqq\left|a_{0}\right|$. Let $\mathscr{D}^{*}=\mathscr{D}-\mathscr{E}_{a}-S$ where $\mathscr{E}_{a}$ is the closed disk with center at the origin and radius $a$, and $S$ is empty or $\{\infty\}$ according to whether or not $\mathfrak{Q}^{\prime}(z)=\boldsymbol{\theta}$. We define a region $\mathscr{D}(z, \zeta)$ to be the union of all points $z$, other than $\zeta$, such that there is a path $\mathscr{P}$ connecting $z$ and $\zeta$ which satisfies the following conditions:

(1) If $\zeta \in \overline{\mathscr{D}}^{*}-\mathscr{D}^{*}, \mathscr{P}$ lies entirely in $\mathscr{D}^{*}$ except for $\zeta$;

(2) For given $\zeta$ and $z$, exp $\left\{q_{21}(\tau)-q_{21}(t)\right\}$ is bounded for all points $t, \tau$ in the order $\zeta, t, \tau, z$ on $\mathscr{P}$. If $q_{1 p}=q_{2 p}$ for $p=0,1, \ldots, r$ and either $q_{1, r+1} \neq q_{2, r+1}$ or $\xi \neq 0$ we also require that $\tau / t$ remain bounded;

(3) $\mathscr{V}_{\mathscr{P}}\left(t^{\eta-1}\right)$ is bounded, where $\eta$ may be taken to be zero if $\xi=0$, otherwise $\eta$ is an arbitrary positive number less than 1 .

By section $3.3 \mathscr{D}(z, \zeta)$ is a domain.

If $q_{21}(z)=q_{2}(z)-q_{1}(z) \neq 0$ (and hence $\xi=0$ ) let

$$
\begin{gathered}
\mathbf{R}_{11 m}(z)=m u_{11 m} z^{-m-1}-\sum_{s=0}^{m-1}\left[b_{11, m-s}(z) u_{11 s}+b_{12, m-s}(z) u_{12}\right] z^{-m-2} \\
\mathbf{R}_{21 m}(z)=q_{21}^{\prime}(z) u_{21 m} z^{-m}-\sum_{k=p+1}^{r+1}\left(q_{2 k-q 1 k}\right) z^{r-k} u_{21 m} z^{-m} \\
-\sum_{\mu=m+p+1}^{m+r+1}\left[\sum_{s=\mu+1-m}^{m i n}\left(q_{2 s}-q_{1 s}\right) u_{21, \mu-s}+(\mu-r-1) u_{21, \mu-r-1}\right. \\
\left.+\sum_{s=0}^{\mu-r-2}\left(b_{11 s} u_{11, \mu-r-2-s}+b_{12 s} u_{21, \mu-r-2-s}\right)\right] z^{r-\mu}-\sum_{s=0}^{m-1}\left[b_{11, m-s}(z) u_{11 s}+b_{12, m-s}(z) u_{21 s}\right] z^{-m-2},
\end{gathered}
$$

where $p$ is the smallest nonnegative integer such that $q_{2 p} \neq q_{1 p}$. On the other hand, if $q_{2}(z)=q_{1}(z)$ then

$$
\begin{aligned}
& \left(\begin{array}{l}
\mathbf{R}_{11 m}(z) \\
\mathbf{R}_{21 m}(z)
\end{array}\right)=\left\{\left[-\left(\begin{array}{ll}
u_{11 m} & u_{12 m} \\
u_{21 m} & u_{22 m}
\end{array}\right)\left(\begin{array}{ll}
0 & 0 \\
\xi & 0
\end{array}\right)+\left(\begin{array}{ll}
m & 0 \\
\xi & m
\end{array}\right)\left(\begin{array}{ll}
u_{11 m} & u_{12 m} \\
u_{21 m} & u_{22 m}
\end{array}\right)\right] z^{-m-1}\right. \\
& \left.\sum_{s=0}^{m-1}\left[\left(\begin{array}{ll}
b_{11, m-s}(z) & b_{12, m-s}(z) \\
b_{21, m-s}(z) & b_{22, m-s}(z)
\end{array}\right)\left(\begin{array}{ll}
u_{11 s} & u_{12 s} \\
u_{21 s} & u_{22 s}
\end{array}\right) z^{-m-2}\right]\right\}\left(\begin{array}{l}
1 \\
\xi \log z
\end{array}\right) .
\end{aligned}
$$

The integral equation for the difference between an actual solution vector and a partial sum of a formal solution vector thus takes the form

$$
\boldsymbol{\epsilon}_{1 m}(z)=\int_{\zeta}^{z} \exp \left[\mathfrak{Q}(z)-\mathfrak{Q}(t)-\mathbf{I}\left(q_{1}(z)-q_{1}(t)\right)\right]\left\{t^{-2} \mathfrak{B}(t) \boldsymbol{\epsilon}_{1 m}(t)-\mathbf{R}_{1 m}(t)\right\} d t
$$

where the path of integration satisfies the above conditions, and $\mathbf{R}_{1 m}(z)=\left(\mathbf{R}_{11 m}(z), \mathbf{R}_{21 m}(z)\right)^{T}$. 
We define nonnegative numbers $u_{11 m}^{*}$ and $u_{21 m}^{*}$ to be the suprema when $\tau \in \mathscr{P}$ of the respective expressions

$$
\left|\frac{\left(u_{11 m}+\xi u_{12 m} \log \tau\right) \tau^{-m}-\left(u_{11 m}+\xi u_{21 m} \log \zeta\right) \zeta^{-m}}{\tau^{\eta_{1}-m}-\zeta^{\eta_{1}-m}}\right|
$$

$\left|\frac{\left(u_{21 m}+\xi u_{22 m} \log \tau\right) \tau^{-m} e^{q_{21}(\tau)-q_{21}(\zeta)} \mid-\left\{u_{21 m}+\xi\left[\log \frac{\tau}{\zeta}\left(u_{11 m}+u_{21 m} \log \zeta\right)+u_{22 m} \log \zeta\right]\right\} \zeta^{-m}}{\tau^{\eta_{1}-m}-\zeta^{\eta_{1}-m}}\right|$

where $\eta_{1}$ may be taken to be zero if $\xi=0$ but otherwise $\eta_{1}$ is an arbitrary positive number less than 1 .

Similarly with $t_{1}$ and $t_{2}$ points on $\mathscr{P}$ in the order $\zeta, t_{1}, t_{2}, z$ we define

$$
\gamma_{11 m}=\sup _{t \in \mathscr{P}}\left|\frac{t^{-\eta_{1}}}{m+1-\eta_{1}} \sum_{s=0}^{m-1}\left\{b_{11, m-s}(t)\left[u_{11 s}+\xi \log t u_{12 s}\right]+b_{12, m-s}(t)\left[u_{21 s}+\xi \log t u_{22 s}\right]\right\}\right|
$$

$\gamma_{21 m}=\sup _{t_{1}, t_{2} \in \mathscr{P}} \mid \frac{p_{21}\left(t_{2}\right) / q_{21}^{\prime}\left(t_{2}\right)-e^{q_{21}\left(t_{2}\right)-q_{21}(\zeta)} p_{21}(\zeta) / q_{21}^{\prime}(\zeta)}{t_{2}^{\eta_{1}-m}-\zeta^{\eta_{1}-m}}+\frac{t^{-\eta_{1}}}{m+1-\eta_{1}}\left[e^{q_{21}\left(t_{2}\right)-q_{21}\left(t_{1}\right)}\left\{-\frac{d}{d t_{1}}\left(\frac{p_{21}\left(t_{1}\right)}{q_{21}^{\prime}\left(t_{1}\right)}\right)\right\} t_{1}^{m+2}\right.$

$\left.+\sum_{s=0}^{m-1} \sum_{k=1}^{2}\left\{\left[b_{2 k, m-s}\left(t_{1}\right)+\xi \log \left(\frac{t_{2}}{t_{1}}\right) \quad b_{1 k, m-s}\left(t_{1}\right)\right]\left[u_{k 1 s}+\xi \log t_{1} u_{k 2 s}\right]\right\}+t_{1}^{m+2} \frac{d}{d t_{1}}\left(\frac{p_{21}\left(t_{1}\right)}{q_{21}^{\prime}\left(t_{1}\right)}\right)\right] \mid$

where

$$
p_{21 m}(t)=\mathbf{R}_{21 m}(t)-q_{21}^{\prime}(t) u_{21 m} t^{-m}+\sum_{s=0}^{m-1}\left[b_{11, m-s}(t) u_{11 s}+b_{12, m-s}(t) u_{21 s}\right] t^{-m-2}
$$

with $\mathbf{R}_{21 m}$ given by (4.12), and $t_{1}, t_{2}$ points on $\mathscr{P}$ in the order $\zeta, t_{1}, t_{2}, z$. Note that $p_{21}(t)=0$ if the smallest integer $p$ such that $q_{2 p} \neq q_{1 p}$ is $r+1$.

We define

$$
\begin{gathered}
(1-\eta) a_{k}=\sup _{t \in \mathscr{P}}\left|t^{-\eta} b_{1 k}(t)\right| \\
(1-\eta) a_{s}=\sup _{t_{1}, t_{2} \in \mathscr{P}}\left|t_{1}^{-\eta}\left[b_{2 k}\left(t_{1}\right)+\xi \log \left(\frac{t_{2}}{t_{1}}\right) b_{1 k}\left(t_{1}\right)\right] e^{q_{21}\left(t_{2}\right)-q_{21}\left(t_{1}\right)}\right| \\
(k=1,2 ; s=k+2) .
\end{gathered}
$$

In (4.18) $t_{1}$ and $t_{2}$ are defined as in (4.16), and $\eta$ may be taken zero if $\xi$ is zero; otherwise $\eta$ is an arbitrary positive number less than 1 . 
Summarizing we have

THEOREM 4.1: If, corresponding to the formal vector solution $\widetilde{\mathbf{W}}_{1}(\mathrm{z})$ of equation (4.3), we can determine a domain $\mathscr{D}(\mathrm{z}, \zeta)$ satisfying the conditions above, then the equation (4.3) possesses an actual solution vector of functions holomorphic in $\mathscr{D}(\mathrm{z}, \zeta)$, given by

$$
\mathbf{W}_{1 \mathrm{~m}}(\mathrm{z})=\left[\begin{array}{c}
\mathbf{W}_{11 \mathrm{~m}}(\mathrm{z}) \\
\mathbf{W}_{21 \mathrm{~m}}(\mathrm{z})
\end{array}\right]=\left[\sum_{\mathrm{k}=0}^{\mathrm{m}-1}\left\{\left(\begin{array}{l}
\mathrm{u}_{11 \mathrm{k}} \\
\mathrm{u}_{21 \mathrm{k}}
\end{array}\right)+\xi \log \mathrm{z}\left(\begin{array}{c}
\mathrm{u}_{12 \mathrm{k}} \\
\mathrm{u}_{22 \mathrm{k}}
\end{array}\right)\right\} \mathrm{z}^{-\mathrm{k}}+\left(\begin{array}{c}
\boldsymbol{\epsilon}_{11 \mathrm{~m}}(\mathrm{z}) \\
\boldsymbol{\epsilon}_{21 \mathrm{~m}}(\mathrm{z})
\end{array}\right)\right] \mathrm{e}^{q_{1}(\mathrm{z})}
$$

where

$$
\left(\begin{array}{l}
\left|\boldsymbol{\epsilon}_{11 \mathrm{~m}}(\mathrm{z})\right| \\
\left|\boldsymbol{\epsilon}_{21 \mathrm{~m}}(\mathrm{z})\right|
\end{array}\right) \leqq \exp \left\{\left(\begin{array}{cc}
\mathrm{a}_{1} & \mathrm{a}_{3} \\
\mathrm{a}_{2} & \mathrm{a}_{4}
\end{array}\right) \mathscr{V}_{\mathscr{P}}\left(\mathrm{t}^{\eta-1}\right)\right\} \times\left\{\begin{array}{l}
\mathrm{u}_{11 \mathrm{~m}}^{*} \mathscr{V}_{\mathscr{P}}\left(\mathrm{t}^{\eta_{1}-\mathrm{m}}\right)+\gamma_{11 \mathrm{~m}} \mathscr{\mathscr { P }}_{\mathscr{P}}\left(\mathrm{t}^{\eta_{1}-m-1}\right) \\
\mathrm{u}_{21 \mathrm{~m}}^{*} \mathscr{V}_{\mathscr{P}}\left(\mathrm{t}^{\eta_{1}-\mathrm{m}}\right)+\gamma_{21 \mathrm{~m}} \mathscr{V}_{\mathscr{P}}\left(\mathrm{t}^{\eta_{1}-m-1}\right)
\end{array}\right\}
$$

for all $\mathrm{z}$ in $\mathscr{D}(\mathrm{z}, \zeta)$. The solution $\mathbf{W}_{1 m}(\mathrm{z})$ depends on $\zeta$ and an arbitrary positive integer $\mathrm{m}$. The function $\mathrm{q}_{1}(\mathrm{z})$ is defined by (4.5); the numbers $\mathrm{u}_{\mathrm{ilm}}^{*}, \gamma_{\mathrm{ilm}}$ and $\mathrm{a}_{1}, \mathrm{a}_{2}, \mathrm{a}_{3}, \mathrm{a}_{4}$ are defined by (4.15), (4.16), and (4.18). If $\xi=0$, both $\eta$ and $\eta_{1}$ may be taken to be zero; otherwise $\eta$ and $\eta_{1}$ are arbitrary positive numbers less than 1 .

The right side of (4.20) may be explicitly evaluated by use of

$$
\begin{gathered}
\exp \left\{\begin{array}{cc}
\alpha & \beta \\
\gamma & \delta
\end{array}\right\}=\left[\frac{\sinh \chi}{\chi}\left(\begin{array}{cc}
\frac{\alpha-\delta}{2} & \beta \\
\gamma & \frac{\delta-\alpha}{2}
\end{array}\right)+\cosh \chi \mathbf{I}\right] e^{1 / 2(\alpha+\delta)} \\
\chi=\frac{1}{2} \sqrt{(\alpha-\delta)^{2}+4 \beta \gamma}
\end{gathered}
$$

\section{The Choice of Paths}

The conditions on the paths of integration in this paper are somewhat weaker and define a larger domain of validity for the asymptotic expansions than the conditions given in [1]. The paths which minimize the error bounds under the present conditions are not known. In application, we suggest that paths similar to those of [5] be tried; the possible loss in accuracy is offset by the greater ease of evaluation of the variations.

Often the error bound can be appreciably reduced by the following simple procedure. Consider for example, the distinct eigenvalue case 1. Let $\mathbf{T}_{0}$ be a matrix such that $\mathbf{T}_{0}^{-1} \mathbf{A}_{0} \mathbf{T}_{0}=\Lambda$ is diagonal. Then $\left(\mathbf{T}_{0} \mathbf{D}\right)^{-1} \mathbf{A}_{0}\left(\mathbf{T}_{0} \mathbf{D}\right)=\Lambda$, where $\mathbf{D}$ is an arbitrary nonsingular diagonal matrix. We choose $\mathbf{D}$ so that the least upper bound of the off-diagonal elements in the resulting coefficient matrix is minimized on the path $\mathscr{P}$. This applies also to the more general case of this paper, except that here we may destroy the similarity of the canonical form to the Jordan canonical form. If we want to preserve the canonical form then for every Jordan leading subblock of $\mathfrak{S}(z)$ of the form

$$
\sum_{k=0}^{r} q_{i k} z^{r-k} \mathbf{1}+q_{i, r+\mathbf{1}} \mathbf{J} \log z
$$

where $\mathbf{J}$ has a full set of units in its first lower diagonal and is zero elsewhere, we must make the corresponding elements of $\mathbf{D}$ equal.

On inspecting the proof of Theorem 2.1, [1], we observe that the undeterminable diagonal elements of $\mathbf{T}_{k}(k=1,2, \ldots, r+1)$ were chosen to be zero. By introducing nonzero diagonal elements we may again be able to improve on the error bounds. A similar observation applies to the zero-inducing transformations used in [3]. 


\section{Summary}

The results of this paper and the preceding paper extend the work of Olver [5] for the secondorder equation to a system of first-order equations. In the majority of applications it is expected that the eigenvalues of the lead coefficient matrix $\mathbf{A}_{0}$ are distinct. The analysis for this case is considerably simpler and different from the analysis for the case when the eigenvalues for the lead coefficient matrix are not distinct, and this is the main reason that the presentation has been split into two parts.

In all cases the procedure was to transform (1.1) to canonical form, to obtain formal solutions for the transformed system, and to obtain actual solutions such that the formal solutions are asymptotic expansions of the actual solutions of the transformed system, as $z \rightarrow \infty$ in some sector of the complex plane. Two cases arise when the eigenvalues of $\mathbf{A}_{0}$ are distinct: the extreme eigenvalue case and the interior eigenvalue case. They are characterized geometrically by the relative location of the eigenvalues of $\mathbf{A}_{0}$ in the complex plane. The main distinction between these two cases is that in the extreme eigenvalue case it is possible to express the error vector - that is, the difference between an actual solution vector and a partial sum of a formal solution vector-by use of a single Volterra vector integral equation, while in the interior eigenvalue case it is necessary to use a simultaneous pair of Volterra vector integral equations. As a consequence, the error bounds are considerably sharper in the extreme eigenvalue case.

When the coefficient matrix in (1.1) is regular for all sufficiently large $z$ and when the eigenvalues of $\mathbf{A}_{0}$ are distinct, it is always possible to choose end-points of integration in the Volterra vector integral equations at infinity. This is no longer the case when the eigenvalues of $\mathbf{A}_{0}$ are not distinct. Further, in this latter case we can no longer always give a simple geometric characterization of extreme and interior eigenvalue cases in terms of the relative location of the eigenvalues of $\mathbf{A}_{0}$. We can, nevertheless, always express the error vector by at most a simultaneous pair of Volterra vector integral equations.

The case $n=2$ is likely to be the most important in applications. The complete solution for this case is given in detail, including a full statement of the transformation to canonical form.

The work of this paper was supported by the Harold Hayward Parlee Memorial Fellowship at the University of Alberta. The author is grateful to Dr. F. W. J. Olver for many valuable criticisms.

\section{References}

[1] Stenger, F., Error bounds for asymptotic solutions of differential equations. I. The distinct eigenvalue case, J. Res. NBS 70B (Math. and Math. Phys.) No. 3. 167 (1966).

[2] Hokuhara, M., Sur les points singuliers des equations differentielles lineares, II of the Fac. of Sci., Hokkaido Imp. Univ., Ser. I, 5, 123-166, (1937).

[3] Turrittin, H. L., Convergent solutions of ordinary differential equations in the neighborhood of an irregular singular point, Acta. Math. 93, 27-66, (1955).

[4] Trjitzinsky, W. J., Analytic theory of linear differential equations, Act. Math. 62, 167-226, (1933).

[5] Olver, F. W. J., On the asymptotic solution of second order differential equations having an irregular singularity of rank 1, with an application to Whittaker functions, J. Soc. Indust. Appl. Math. Num. Anal. Ser. B, 225-243 (1965).

[6] Thorne, R. C., Asymptotic formulae for solutions of linear second order differential equations with large parameter, Australian Math. Soc. 1, 439-464, (1960).

[7] Stenger, F., Error bounds for solutions of differential equations, Ph.D. thesis, University of Alberta, Edmonton (1965).

[8] Collatz, L., Funktionalanalysis und Numerische Mathematik, Springer-Verlag (1964).

(Paper 70B3-181) 